\title{
MECP2 Duplication Causes Aberrant GABA Pathways, Circuits and Behaviors in Transgenic Monkeys: Neural Mappings to Patients with Autism
}

\begin{abstract}
Dan-Chao Cai, ${ }^{1 *}$ Zhiwei Wang, ${ }^{1,2^{*}}$ Tingting Bo, ${ }^{1,2^{\star}}$ Shengyao Yan, ${ }^{1,2^{*}}$ Yilin Liu, ${ }^{1,2}$ Zhaowen Liu, ${ }^{3,4}$ ${ }^{\circ}$ Kristina Zeljic, ${ }^{1,2}{ }^{\circledR}$ Xiaoyu Chen, ${ }^{1,2}$ Yafeng Zhan, ${ }^{1,2}$ Xiu Xu, ${ }^{5}$ Yasong Du, ${ }^{6}$ Yingwei Wang, ${ }^{7}$ Jing Cang, ${ }^{8}$ Guang-Zhong Wang, ${ }^{9}$ Jie Zhang, ${ }^{4}$ Qiang Sun, ${ }^{1}$ Zilong Qiu, ${ }^{1}$ Shengjin Ge, ${ }^{8}{ }^{\circledR}$ Zheng Ye, ${ }^{1,2}$ and ${ }^{\circledR}$ Zheng Wang ${ }^{1,2,10}$ ${ }^{1}$ Institute of Neuroscience, CAS Center for Excellence in Brain Science and Intelligence Technology, State Key Laboratory of Neuroscience, CAS Key Laboratory of Primate Neurobiology, Chinese Academy of Sciences, Shanghai 200031, People's Republic of China, ${ }^{2}$ University of Chinese Academy of Sciences, Shanghai 200031, People's Republic of China, ${ }^{3}$ Life Science Research Center, Engineering Research Center of Molecular and Neuro Imaging of Ministry of Education, School of Life Science and Technology, Xidian University, Xi'an 710126, People's Republic of China, ${ }^{4}$ Institute of Science and Technology for Brain Inspired Intelligence, Key Laboratory of Computational Neuroscience and Brain Inspired Intelligence, Fudan University, Shanghai 200433, People's Republic of China, ${ }^{5}$ Department of Child Healthcare, Children's Hospital of Fudan University, Shanghai 201102, People's Republic of China, ${ }^{6}$ Shanghai Mental Health Center, Shanghai Jiao Tong University School of Medicine, Shanghai 200030, People's Republic of China, ${ }^{7}$ Department of Anesthesiology, Huashan Hospital of Fudan University, Shanghai 200040, People's Republic of China, ${ }^{8}$ Department of Anesthesiology, Zhongshan Hospital of Fudan University, Shanghai 200032, People's Republic of China, ${ }^{9}$ Key Laboratory of Computational Biology, Chinese Academy of Sciences-Max Planck Partner Institute for Computational Biology, Shanghai Institutes for Biological Sciences, Chinese Academy of Sciences, Shanghai 200031, People's Republic of China, and ${ }^{10}$ Kunming Institute of Zoology, Chinese Academy of Sciences, Kunming 650223, People's Republic of China
\end{abstract}

MECP2 gain-of-function and loss-of-function in genetically engineered monkeys recapitulates typical phenotypes in patients with autism, yet where MECP2 mutation affects the monkey brain and whether/how it relates to autism pathology remain unknown. Here we report a combination of gene-circuit-behavior analyses including MECP2 coexpression network, locomotive and cognitive behaviors, and EEG and fMRI findings in 5 MECP2 overexpressed monkeys (Macaca fascicularis; 3 females) and 20 wild-type monkeys (Macaca fascicularis; 11 females). Whole-genome expression analysis revealed $M E C P 2$ coexpressed genes significantly enriched in GABA-related signaling pathways, whereby reduced $\beta$-synchronization within fronto-parieto-occipital networks was associated with abnormal locomotive behaviors. Meanwhile, MECP2-induced hyperconnectivity in prefrontal and cingulate networks accounted for regressive deficits in reversal learning tasks. Furthermore, we stratified a cohort of 49 patients with autism and 72 healthy controls of 1112 subjects using functional connectivity patterns, and identified dysconnectivity profiles similar to those in monkeys. By establishing a circuit-based construct link between genetically defined models and stratified patients, these results pave new avenues to deconstruct clinical heterogeneity and advance accurate diagnosis in psychiatric disorders.

Key words: autism spectrum disorder; brain connectome; cognitive flexibility; MECP2 overexpression; repetitive restricted behavior; transgenic monkeys

Significance Statement

Autism spectrum disorder (ASD) is a complex disorder with co-occurring symptoms caused by multiple genetic variations and brain circuit abnormalities. To dissect the gene-circuit-behavior causal chain underlying ASD, animal models are established by manipulating causative genes such as MECP2. However, it is unknown whether such models have captured any circuit-level pathology in ASD patients, as demonstrated by human brain imaging studies. Here, we use transgenic macaques to examine the causal effect of $M E C P 2$ overexpression on gene coexpression, brain circuits, and behaviors. For the first time, we demonstrate that the circuit abnormalities linked to MECP2 and autism-like traits in the monkeys can be mapped to a homogeneous ASD subgroup, thereby offering a new strategy to deconstruct clinical heterogeneity in ASD. 


\section{Introduction}

Psychiatric disorders including autism spectrum disorder (ASD) are increasingly prevalent, and substantially heterogeneous in genetic bases and phenotypic architecture, consisting of subtypes with distinct biological mechanisms (Lombardo et al., 2019). To achieve precise diagnosis and treatment, patient stratification based on biological measures has been encouraged in recent research initiatives (Lombardo et al., 2019). Using noninvasive electrophysiological and neuroimaging measures, neural subtypes or biotypes of mental illnesses have been identified based on distinct patterns of brain network dysfunction, which are disease relevant and predictive of treatment responsiveness (Drysdale et al., 2017). Recent efforts have also been made to bring genetic disorders with a high penetrance of ASD to the forefront of translational efforts to find treatments for subpopulations of mechanism-based classification of ASD (Sahin and Sur, 2015). Although this strategy holds significant potential in filtering the complex etiology of autism, how specific genes with rare copy number variants contribute to functional alterations in brain circuitry, and what neural circuit basis may underlie particular pathologic behaviors in ASD remains elusive (Sahin and Sur, 2015). This line of research has probably been held back by the low occurrence of rare and de novo copy number variants in individuals with ASD (Huguet et al., 2013).

An alternative route to dissect clinical heterogeneity and pursue the underlying neuropsychiatric mechanisms is genetic animal modeling, where certain causative genes in the biologically homogeneous samples are purposely manipulated to recapitulate some dimensions of core symptoms in psychiatric patients (van den Heuvel et al., 2016). One could expect that such animal models with a clear genetic basis would (at least partially) share circuit constructs with certain psychiatric neural subtypes, thereby providing valuable opportunities for probing the genecircuit-behavior casual chain of events underlying complex brain disorders (van den Heuvel et al., 2016; Bertero et al., 2018). As extensively demonstrated in both humans (Ramocki et al., 2010) and rodent models (Samaco et al., 2012), methyl-CpG binding protein 2 (MECP2) is one of a few exceptional genes of causal effect established in ASD (Monteggia et al., 2018). Mutations and duplications of MECP2 lead to Rett syndrome (Amir et al., 1999) and MECP2 duplication syndrome (Ramocki et al., 2010), respectively. Both exhibited a wide range of detrimental behavior effects including motor impairments, stereotyped behaviors, anxiety, social avoidance, and other neuropsychiatric symptoms. Using genetically manipulated rodent models, researchers have demonstrated that both loss and gain of MECP2 function can alter synaptic transmission and disrupt the overall excitation/inhibition balance in neural circuits (Guy et al., 2011; Lu et al., 2016). We have recently reported the successful application of lentiviralmediated methods to produce genetically engineered macaque

This work was supported by the National Key R\&D Program of China (Grants 2017 YFC1310400 and 2018YF(1313803); the Strategic Priority Research Program of Chinese Academy of Science (Grant XDB32000000); the National Natural Science Foundation (Grants 81571300, 81527901, 81730031, and 31771174); Natural Science Foundation and Major Basic Research Program of Shanghai (Grant 16JC1420100); Shanghai Municipal Science and Technology Major Project (Grant 2018SHZDZX05); and Key Realm R\&D Program of Guangdong Province (Grant 2019B030335001). We thank Hu Zhang, Ganxian Wang, Qinying Jiang, and Wenwen Yu for assistance to monkey training and data acquisition; and Drs. Trevor Robbins, Freund Tamas, Charles Schroeder, Anna Roe, and Muming Poo for stimulating discussions and suggestions during the preparation of this study.

Correspondence should be addressed to Zheng Wang at zheng.wang@ion.ac.cn or Shengjin Ge at ge. shengjin@zs-hospital.sh.cn.

https://doi.org/10.1523/JNEUROSCI.2727-19.2020

Copyright $\odot 2020$ the authors monkeys carrying extra copies of MECP2 that manifested less active social contact and increased stereotypical behaviors (Liu et al., 2016). However, the relevant brain circuits that mediate the causal effects of atypical MECP2 levels on phenotypic symptoms in transgenic (TG) monkeys remain essentially unexplored. In addition, it is unknown whether the disrupted circuit observed in the monkey model would map neatly onto homologs in specific neural subtypes of autistic patients.

To address these questions, we use a combination of MECP2 coexpression network analysis, locomotive and cognitive behavioral tests (Buckley et al., 2009; Judge et al., 2011), resting-state fMRI (Neubert et al., 2014; van den Heuvel et al., 2016), and EEG recordings (Gil-da-Costa et al., 2013), all of which are commonly administered in primate species and are potentially amenable to cross-species translation into clinical diagnosis and future development of therapeutic interventions for autismrelated disorders. We further conduct a cross-species circuit mapping of dysfunctional connectivity profiles between transgenic monkeys and subgroups of ASD patients who are stratified based on whole-brain resting-state functional connectivity patterns.

\section{Materials and Methods \\ Participants}

Five MECP2-overexpressed TG monkeys (Macaca fascicularis; three female) and 20 wild-type (WT) monkeys (Macaca fascicularis; 11 female) participated in the present study. All TG monkeys participated in all three behavioral tasks. Subgroups of 16, 7, and 4 WT monkeys participated in home cage observation, peer separation tests, and reversal learning tasks, respectively. All TG and 16 WT monkeys participated in the EEG experiment. All TG and 11 WT monkeys participated in the MRI experiment. In addition, the transcriptome data from two previously published studies were reanalyzed (Bernard et al., 2012; Liu et al., 2016).

\section{Ethical statement}

All experimental procedures for nonhuman primate research in this study were approved by the Institutional Animal Care and Use Committee at the Institute of Neuroscience and the Biomedical Research Ethics Committee, Shanghai Institutes for Biological Sciences, Chinese Academy of Sciences, and conformed to National Institutes of Health guidelines for the humane care and use of laboratory animals.

\section{Weighted gene coexpression network analysis}

To gain system-level insight into how MECP2 mutations in TG monkeys affect biological processes, we first performed a weighted gene coexpression network analysis (WGCNA) to construct the coexpression network of $M E C P 2$ in the macaque brain. We obtained gene expression data for the whole neocortex of three male and three female adult rhesus macaques (Macaca mulatta) published from previous work (Bernard et al., 2012). We performed quality control processing for the raw expression data, including exclusion of outlier samples with interarray correlations (IACs) $<2$ SDs from the mean IAC, and correction for the cross-batch with the ComBat package in R (Johnson et al., 2007). Furthermore, we obtained the expression value of genes from their corresponding probes and selected the probe with the highest variation across samples to represent this gene. Finally, we included expression profiles of 13,888 genes in 182 samples in the following analysis (Fig. 1A).

The WGCNA algorithm using a dynamic cut-tree method was used to produce gene coexpression clusters using the WGCNA package in $\mathrm{R}$ (Langfelder and Horvath, 2008). A MECP2 coexpression network was defined as genes within the same module as MECP2 and significantly correlated with the module eigengene (ME). Furthermore, we performed functional enrichment analysis using the Toppgene suite (https:// toppgene.cchmc.org) to infer biological processes related to the MECP2 coexpression network. We also compared the expression level of MECP2 

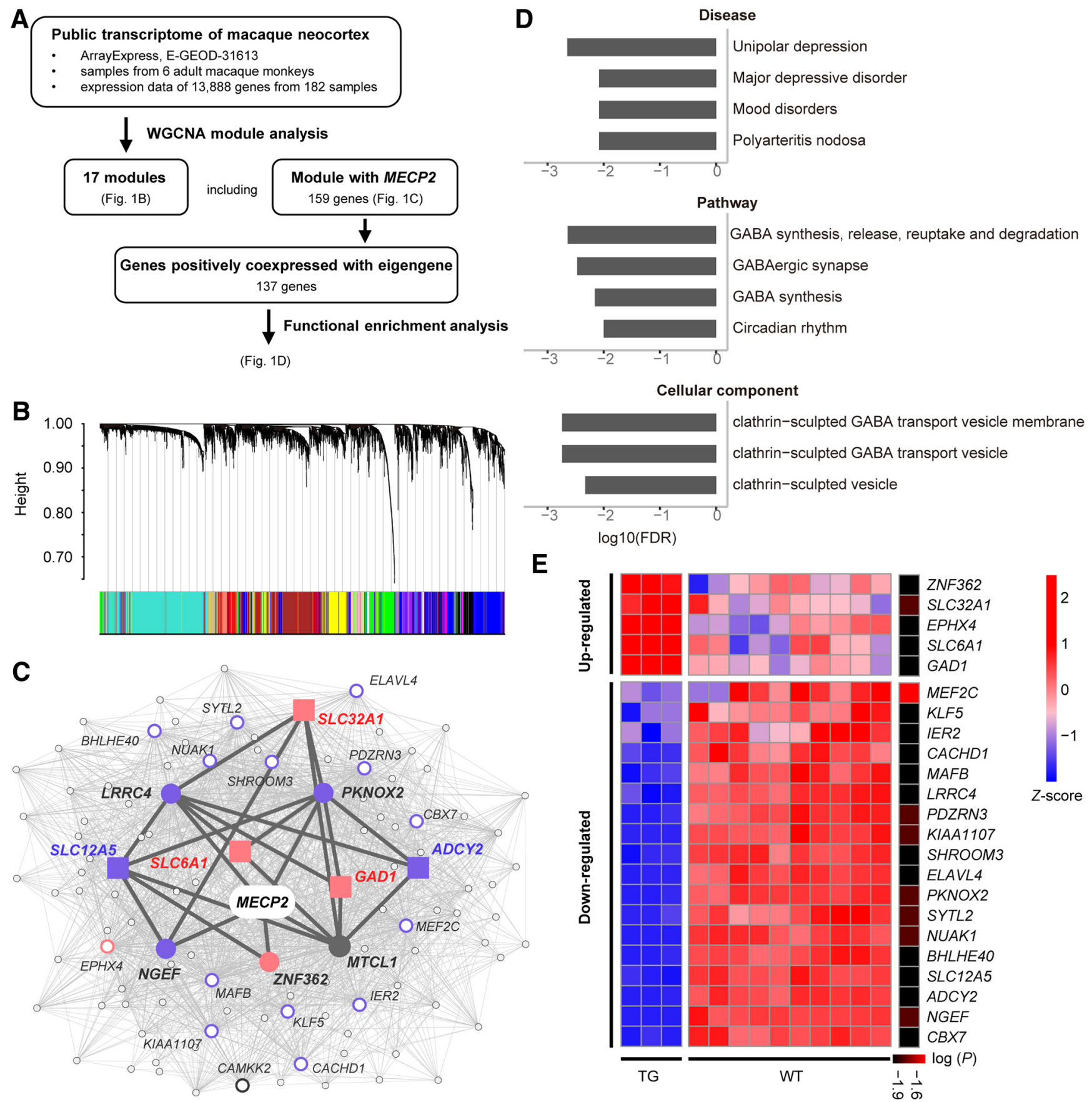

\section{E}
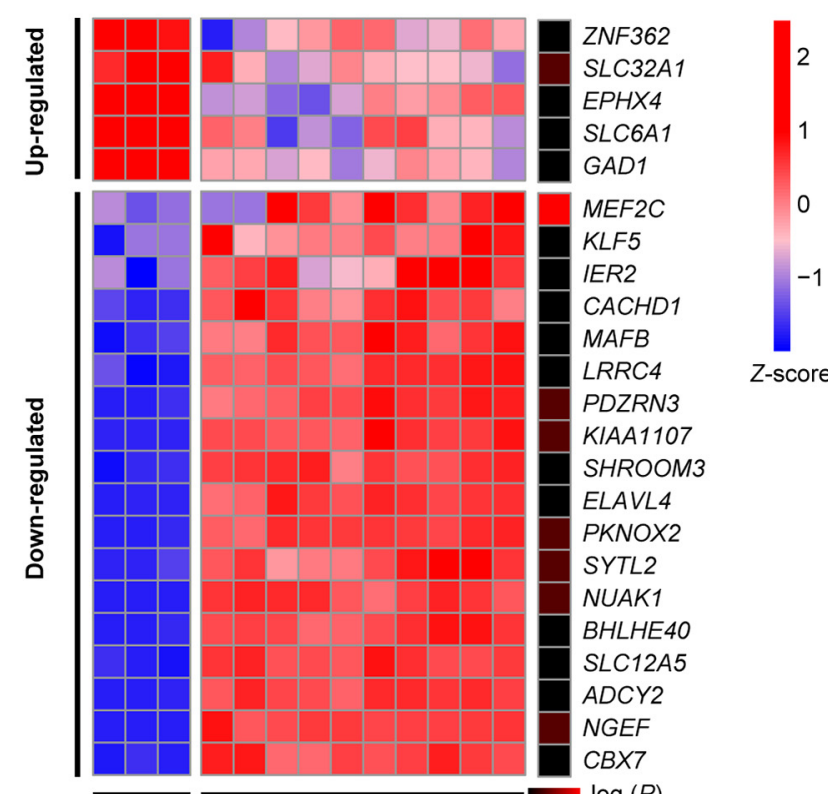

KIAA1107

SHROOM 3

ELAVL4

PKNOX2

SYTL2

NUAK1

BHLHE40

SLC12A5

$A D C Y 2$

NGEF

$C B X 7$

TG

$\log (P)$

Figure 1. WGCNA and functional enrichment analysis of MECP2-coexpressed genes. A, Overview of the weighted gene coexpression network analysis using public transcriptional data of 182 macaque cortical samples, and functional enrichment analysis on identified gene module coexpressed with MECP2. B, Network analysis dendrograms representing assignment of 13,888 genes to 17 modules. C, MECP2 coexpression network. Filled circles and squares indicate directed neighbors of MECP2 $(n=5)$ and genes enriched in GABA-related pathways $(n=5)$, respectively. All other second-order neighbors of MECP2 are represented with empty circles, where larger circles indicate the top 20 genes with larger node degree. Pink and purple colors indicate upregulation and downregulation in MECP2 transgenic monkeys, respectively. Dark gray lines indicate the path from MECP2 to GABA-associated genes. $D$, Mood disorders, four gene pathways, and three cellular components are significantly enriched in constrained genes (FDR-corrected, $p<0.01$ ). E, Heatmap for the expression of MECP2-correlated genes in the cortical regions of $M E C P 2$ transgenic monkeys. Significant level $[\log (p)]$ is indicated in the sidebar. Red and blue colors indicate high and low expression, respectively.

coexpressed genes in three deceased TG and four WT monkeys using a previously published transcriptome of cortical samples (Liu et al., 2016).

\section{Monkey behavioral tasks}

Locomotive behavior in home cage

$M E C P 2$-overexpressed monkeys in late infancy and early juvenile stages (12 18 months) exhibited an increased frequency of repetitive circular locomotion (Liu et al., 2016). When they reached $\sim 55$ months of age, which is equivalent to adolescence in humans (age, 12 18 years; Liu et al., 2015), we repeated the analysis on spontaneous locomotive behaviors in five TG monkeys (mean age, $4.66 \pm 0.08$ years; 3 females) and $16 \mathrm{WT}$ monkeys (mean age, $5.43 \pm 1.05$ years; 7 females) monkeys. Monkeys were individually caged (length, $0.9 \mathrm{~m}$; width, $0.8 \mathrm{~m}$; height, $0.9 \mathrm{~m}$ ) in a room with 10 to 12 roommates. For each monkey, uninterrupted recordings were filmed for $20 \mathrm{~min}$ daily between 2:40 P.M. and 3:00 P.M. on 4 separate days. Behaviors of repetitive circular and general locomotion were analyzed by two independent trained observers who were blinded to the genotypes of the monkeys. Interobserver reliability was evaluated. 

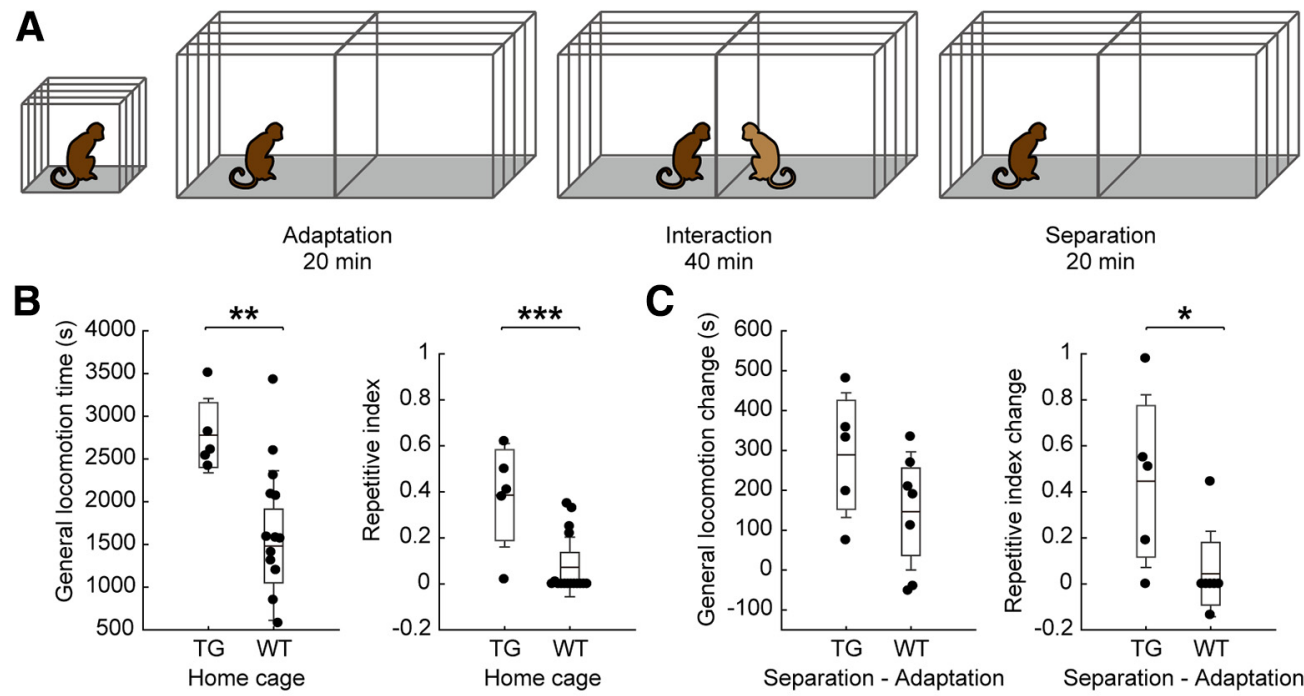

C
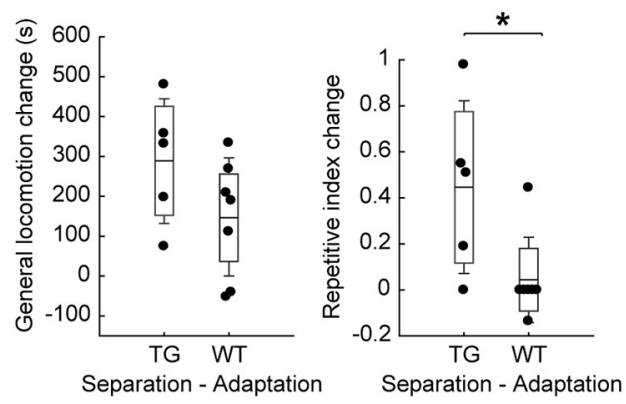

D
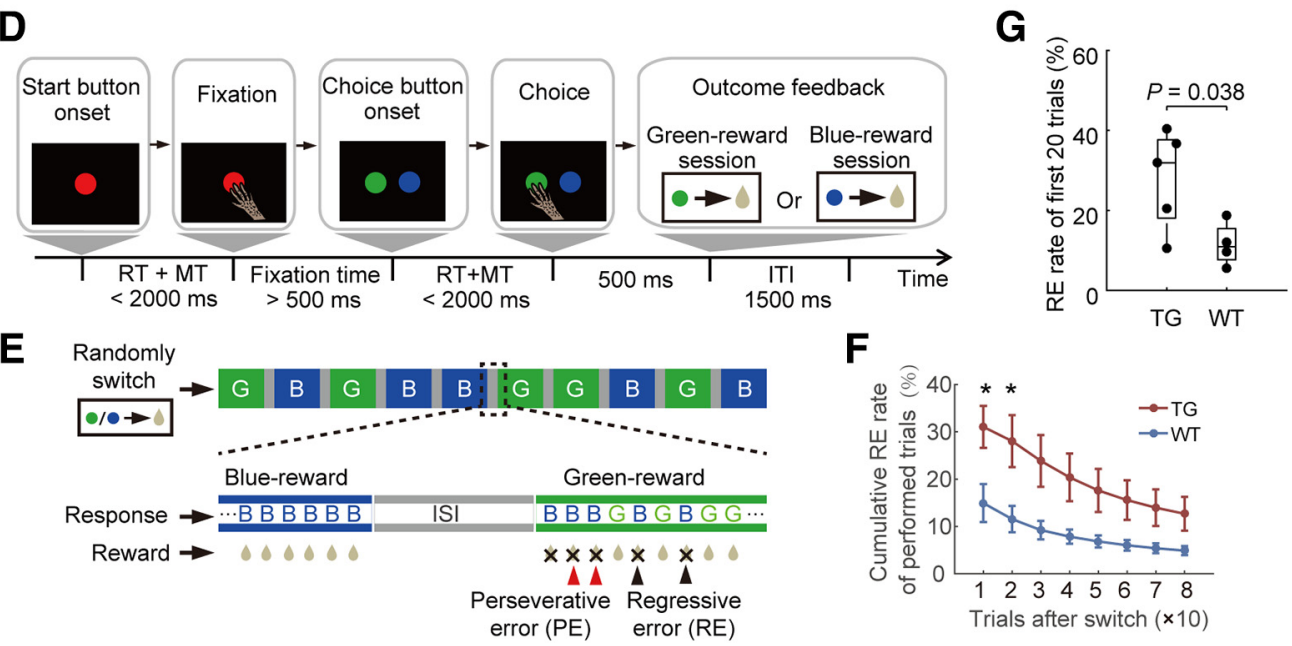

Figure 2. Abnormal locomotive and cognitive flexibility in MECP2 monkeys. $A$, Peer separation paradigm. The test monkey was able to have visual, auditory, and olfactory interactions with the peer monkey during the interaction stage. $\boldsymbol{B}$, Excessive locomotion during home cage observation in TG monkeys in terms of general locomotion time (left) and repetitive index (right). $\boldsymbol{C}$, Larger induced locomotion change in TG monkeys after peer separation. ${ }^{* *} p<0.0001,{ }^{* *} p<0.001,{ }^{*} p<0.05$. D. Temporal sequence of events in the color discrimination and reversal learning task. RT, Reaction time; MT, movement time; ITI, intertrial interval. $E$, An example of task sequence in the reversal learning test. The color assigned for reward was pseudorandomly switched between sessions. G, Green-reward session; B, blue-reward session. $\boldsymbol{F}$, The cumulative regressive error rate of performed trials after rule switch was analyzed ( $n=5$, TG; $n=4$, WT; ${ }^{*} p<0.05$, two-sample Student's $t$ test). Error bars denote SEM. G, The percentage of regressive errors in TG and WT groups in the first 20 performed trials after rule switch (Student's $t$ test).

Repetitive circular locomotion was defined as circular routing, tumbling, and cyclic routing in other more complex paths (e.g., the path of a figure eight) for more than three cycles. General locomotion was defined as any form of locomotion occurring during nonresting states, with resting state referring to staying still for $>3 \mathrm{~s}$ without obvious body movement (e.g., standing, sitting, and grooming). To quantify the repetitive behavior, a repetitive index was defined by dividing repetitive locomotion time by general locomotion time.

Locomotive behavior in peer separation test

TG monkeys also exhibited increased anxiety at an early age in addition to excessive stereotyped locomotion (Liu et al., 2016). To examine the relationship between abnormal locomotion and trait anxiety, we designed a behavioral test (Fig. 2A) based on a classical peer separation paradigm (McKinney et al., 1972). The absolute change of locomotive measures after separation with peers was estimated to indicate the locomotive response to separation anxiety in five TG monkeys (age, $6.50 \pm 0.13$ years; three females) and seven WT monkeys (age, $10.06 \pm 3.79$ years; four females). A test monkey and a peer monkey were used in the paradigm (Fig. 2A). Each of the test monkeys was paired with two familiar and two unfamiliar WT monkeys on four separate sessions. Familiar peers had been housed in the same room as the test monkey for more than 1 year. The test apparatus was a two-compartment cage (length, $3.4 \mathrm{~m}$; width, $1.9 \mathrm{~m}$; height, $1.7 \mathrm{~m}$ ) made of steel rods. These two compartments were separated by electrochromic switchable glass, which turns opaque when a voltage is applied. Testing was conducted from 3:00 P.M. to 5:30 P.M. and consisted of three periods (Fig. 2A). First, the test monkey was transferred to the testing compartment and stayed alone for $20 \mathrm{~min}$. Then the peer monkey was transferred to the other compartment. Two monkeys were able to have visual, auditory, and olfactory contact during a $40 \mathrm{~min}$ interaction period, and both monkeys were recorded on video. Afterward, the peer monkey was removed from the compartment. And then the test monkey was observed alone for a $20 \mathrm{~min}$ period of separation. The glass between two compartments remained transparent for the adaptation and interaction periods. For each monkey, two measures of behavioral patterns (general locomotion and repetitive index) were compared between two groups under two conditions (familiar and unfamiliar).

\section{Color discrimination and reversal learning task}

To probe potential cognitive inflexibility induced by MECP 2 overexpression (Geurts et al., 2009), we trained five TG monkeys (age, $6.68 \pm 0.11$ years; three females) and four WT monkeys (age, 6.33 \pm 0.25 years; two females) to perform a color discrimination and reversal learning task on a touch screen (Buckley et al., 2009; Judge et al., 2011). All monkeys 
were experimentally naive and provided with a standard primate diet except on behavioral test days, before which they were fasted for $24 \mathrm{~h}$. During experiments, the monkey was seated comfortably in a behavioral primate chair without head fixation. The right arm of the monkey could move freely to touch a 19 inch touch screen (1991L open-frame LCD touch screen, Elo). The experimental task was programmed using the MonkeyLogic toolbox (Asaad and Eskandar, 2008). A juice reward was accurately supplied by a peristaltic pump (BT100-1F, Longer Precision Pump), depending on the outcome of each trial. We set up the reversal learning task with three stages.

Stage 1: stimulus-directed touching. Each trial began with a red fixation circle presented in the screen center with a field angle of $2^{\circ}$. The animal needed to touch the fixation circle within $2 \mathrm{~s}$ to receive a reward. Greater reward was received for a longer touch. The maximum reward time was $1 \mathrm{~s}$ in each trial. Animals completed four sessions per day and 200 trials per session, with an intertrial interval of $2 \mathrm{~s}$. The number of days taken to achieve an $80 \%$ correct fixation $(>1 \mathrm{~s})$ within a single session was recorded.

Stage 2: color discrimination. After the animal maintained a $500 \mathrm{~ms}$ fixation on the red fixation circle, a green and a blue circle appeared simultaneously on the screen, one on the left side and the other on the right side. The monkey needed to touch the rewarded color within $2 \mathrm{~s}$ to receive a $500 \mathrm{~ms}$ reward. The position of the rewarded color was randomized. The rewarded color was green for the first 2 weeks and switched to blue afterward. Monkeys performed four sessions per day and 200 trials per session, with an intertrial interval of $1.5 \mathrm{~s}$. Performance in each session was defined as the ratio of correct trials to total trials. The average reaction time in correct trials was also calculated. The number of sessions taken to achieve an $80 \%$ correct rate within a single session after rule switch was recorded.

Stage 3: reversal learning. The rewarded color (green or blue) in the discrimination task was randomly switched between sessions (Fig. 2D,E, Movie 1). No cue was provided to indicate the change of rewarded color. Animals had to discover the currently rewarded color through trial and error. At the beginning of this stage $(\sim 2$ weeks), monkeys performed four sessions per day, as in stage 2, to reinforce reversal learning. In the final test phase ( $\sim 2$ weeks), each monkey was tested with 10 sessions per day and 80 trials per session. The intersession interval was $\sim 1 \mathrm{~min}$. The percentage of correct trials in every 10 trials performed after each rule switch was analyzed. Two types of errors after rule switches were assessed. Perseverative errors were trials in which monkeys continued to select the previously rewarded color following negative feedback but before the first correct response, suggesting a failure to switch to a new rule. By contrast, regressive errors were trials in which monkeys continued to select the previously rewarded color after the first correct response, representing a preference for the old rule or an inability to maintain a new rule (Fig. $2 E$ ).

\section{Animal preparation}

For both resting-state EEG recording and fMRI scanning, animals were prepared and maintained in a stable brain state under light anesthesia. The animal preparation procedure was conducted as described in our

\section{TG08}

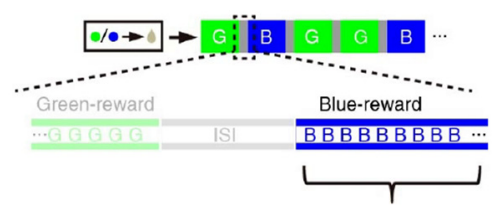

\section{Switch from green-reward session to blue-reward session (First 20 trials)}

Movie 1. An example of monkey (TG08) performing reversal learning task. Last 5 trials in a green-reward session and first 20 trials after a switch to a blue-reward session were recorded. The video is shown at twice the real time speed. [View online] previous work (Lv et al., 2016). Induction of anesthesia was achieved by intramuscular injection with midazolam $(0.25 \mathrm{mg} / \mathrm{kg}$; Nhwa Pharma Co, Ltd.) before EEG recording or with ketamine $(10 \mathrm{mg} / \mathrm{kg}$; Gutian Pharma Co, Ltd.) before MRI scanning, supplemented with atropine sulfate $(0.05 \mathrm{mg} / \mathrm{kg}$; Shanghai Harvest Pharma Co, Ltd.) to decrease bronchial and salivary secretions. After intubation, animals were ventilated with a mixture of isoflurane (2-2.5\%; Lunan Pharma Co, Ltd.) and oxygen via either a standard ventilator (CWE) outside the scanner room or an MRI-compatible ventilator (CWE) inside the scanner room. Macaques were maintained with intermittent positive-pressure ventilation to ensure a constant respiration rate (25-35 breaths/min). The concentration of isoflurane was adjusted based on continuously monitored vital signs, including blood oxygenation, electrocardiogram (EKG), rectal temperature (Small Animal Instruments), respiration rate, and end-tidal $\mathrm{CO}_{2}$ (Smiths Medical ASD). Oxygen saturation was kept $>95 \%$, and body temperature was kept constant using a heated water blanket (Gaymar Industries). Lactated Ringer's solution was given with a maximum rate of $10 \mathrm{ml} / \mathrm{kg} / \mathrm{h}$ during the anesthesia process.

Animals were further prepared with an MRI-compatible EEG cap to record EEG signals and to monitor the brain state during the experiment. The preparation procedure was similar to our previous work (Zhang et al., 2019). The animal scalp was thoroughly cleaned after hair removal. A custom EEG cap made of stretchable materials was fitted over the scalp. An EKG electrode was attached on the back close to the heart to facilitate offline removal of cardioballistic artifacts. Electrode impedance was kept at $<5 \mathrm{k} \Omega$. After setting up the EEG cap, animals were restrained within a water blanket in a sphinx-like position with the head protruding and facing forward. For fMRI scanning, the animal head was further secured using a custom-built MRI-compatible stereotaxic frame after local anesthetic (5\% lidocaine cream) was applied to the ears. Note that EEG data analyzed in the current study were acquired outside the scanner in a separate experiment. EEG data recorded inside the scanner were used to monitor evident EEG signatures related to anesthesia depth, such as burst suppression and paradoxical excitation (Brown et al., 2010). Because of unexpected noise contamination in some data, we did not conduct further analysis on simultaneous EEG data.

\section{Anesthesia maintenance}

In the light of anesthesiologist instructions (Y.W., J.C., and S.G.), anesthesia was maintained using the lowest possible concentration of isoflurane gas during data acquisition. Isoflurane was selected for anesthesia maintenance as numerous studies have demonstrated that stable neural activity and functional connectivity patterns under a narrow range of medium level isoflurane (e.g., $\pm 0.25 \%$ ) are suitable for anesthetized nonhuman primate investigations (Vincent et al., 2007; Shen et al., 2012; Hutchison et al., 2013; Wang et al., 2013). The concentration of isoflurane was adjusted based on both vital signs and EEG signatures. Neither paradoxical excitation nor burst suppression, indicating minimally conscious and deep anesthesia states, respectively, was observed during data collection (Brown et al., 2010). Slight differences in anesthetic gas concentrations were needed to impose a similar level of physiological anesthesia on different monkeys (Sallet et al., 2013). The mean concentration of inhaled isoflurane was $1.26 \pm 0.22 \%$ and $1.12 \pm 0.13 \%$ during EEG recording and fMRI scanning, respectively. Within the range of isoflurane levels used in the current study, consistent patterns of functional coupling between distant brain areas have been reported in prior monkey fMRI studies (Vincent et al., 2007; Hutchison et al., 2014) and demonstrated in our work as well (Wang et al., 2013; Lv et al., 2016).

\section{Monkey EEG data acquisition}

We conducted multichannel scalp EEG recordings in 5 TG monkeys (age, $5.60 \pm 0.08$ years; 3 females) and 16 WT monkeys (age, $5.66 \pm 0.60$ years; 7 females). Spontaneous neural activity was collected from anesthetized monkeys in a shielded chamber room. EEG scalp recordings were acquired with the BrainVision Recorder software using a BrainAmp MR amplifier and a 28-channel EEG cap customized for macaques with sintered $\mathrm{Ag} / \mathrm{AgCl}$ ring electrodes (Brain Products). The spatial distributions of electrodes over the brain were obtained in high-resolution T2-weighted MRI images using a 3D turbo-spin-echo sequence (TR, $3000 \mathrm{~ms}$; TE, $370 \mathrm{~ms}$; field of 
view, $128 \times 88 \mathrm{~mm}$; acquisition voxel size, $0.5 \times 0.5 \times 0.5 \mathrm{~mm}^{3}$; 30 sagittal slices), as demonstrated in Figure $3 A$. Twenty-one active electrodes were projected from scalp to cortex (Fig. $3 B$ ) and labeled according to the closest cortical area. Six recording channels far from the cortex were disabled for subsequent recording. The ground electrode was located far away from the cortex beneath the left eye. The EEG signal was sampled at $5000 \mathrm{~Hz}$ with a resolution of $0.5 \mu \mathrm{V}$ per bit and a measuring range of $\pm 16 \mathrm{mV}$. EEG measurement was repeated in five TG monkeys on different days, and a total of 11 sessions were acquired. For each recording session, 6 to 10 runs lasting 7 min each were collected depending on the physiological state of the animal. Visual inspection was meticulously conducted after EEG preprocessing. Runs with residual artifacts due to incomplete artifact removal or other idiographic artifacts were excluded from further analysis. A total of 76 TG and 102 WT datasets were included in the final analysis.

\section{Monkey MRI data acquisition}

We acquired whole-brain MRI data in 16 anesthetized macaques including 5 TG monkeys (age, $4.40 \pm 0.29$ years; 3 females) and 11 WT monkeys (age, $4.68 \pm 0.46$ years; 7 females). MRI images of monkeys were acquired at the Institute of Neuroscience on a $3 \mathrm{~T}$ whole-body scanner (Trio, Siemens Healthcare) running with an enhanced gradient coil insert (AC88; $80 \mathrm{mT} / \mathrm{m}$ maximum gradient strength, $800 \mathrm{mT} / \mathrm{m} / \mathrm{s}$ maximum slew rate). A custom-built eight-channel phased-array transceiver coil was used for animal imaging. Whole-brain resting-state fMRI data were collected using a gradient-echo EPI sequence [TR, $2000 \mathrm{~ms}$; TE, $29 \mathrm{~ms}$; flip angle, $77^{\circ}$; slices, 32 ; matrix, $64 \times 64$; field of view, $96 \times 96 \mathrm{~mm}$; $1.5 \times 1.5$ $\mathrm{mm}^{2}$ in-plane resolution; slice thickness, $2.5 \mathrm{~mm}$; GRAPPA (generalized, autocalibrating, partially parallel acquisitions) factor, 2]. For each session, 5-10 runs were acquired, and each run consisted of 200 functional volumes. The number of runs depended on the physiological state of the animal. A pair of gradient echo images (TE, 4.22 and $6.68 \mathrm{~ms}$ ) with the same orientation and resolution as EPI images were acquired to generate a field map for distortion correction of EPI images. High-resolution T1-weighted anatomic images were acquired using an MPRAGE sequence (TR, $2500 \mathrm{~ms}$; $\mathrm{TE}, 3.12 \mathrm{~ms}$; inversion time, $1100 \mathrm{~ms}$; flip angle, $9^{\circ}$; acquisition voxel size, $0.5 \times 0.5 \times 0.5 \mathrm{~mm}^{3}$; 144 sagittal slices). Six whole-brain anatomic volumes were acquired and further averaged for better brain segmentation and $3 \mathrm{D}$ cortical reconstruction. Runs showing erratic vital signs or image artifacts were excluded from further analysis. The final analysis included a total of 45 runs from TG monkeys and 99 runs from WT monkeys.

\section{Human MRI data}

The human MRI data were obtained from the Autism Brain Imaging Data Exchange (ABIDE), which shares MRI scans and phenotypic information from 539 patients with ASD and 573 typically developing control subjects (TDCs; Di Martino et al., 2014). To reduce the heterogeneity within human ASD, we conducted screening based on basic demographic and diagnostic information provided in the ABIDE database (Di Martino et al., 2014). We screened human data based on the following two principles: (1) to make the data as homogenous as possible and (2) to make the human data as well matched with the monkey data as possible. The inclusion criteria for patients were as follows: (1) righthandedness, (2) age between 12 and 18 years, (3) a full-scale IQ (FIQ) score between 80 and 130, (4) a diagnosis of autism rather than Asperger syndrome or Pervasive Development Disorder Not Otherwise Specified, according to the Diagnostic and Statistical Manual of Mental Disorders, fourth edition (text revision), and (5) available site-matched TDCs. Finally, the data from nine sites were subject to subsequent analysis, which included 90 adolescent individuals with autism (hereafter, we use autism rather than ASD to refer to this patient cohort; age, $14.75 \pm 1.79$ years; 12 females) and 140 TDCs (age, $14.68 \pm 1.69$ years; 31 females) matched for age, handedness, FIQ, and data source site.

\section{EEG data analysis}

EEG data were preprocessed using the toolbox of EEGLAB (Delorme and Makeig, 2004). Bad channels were identified semiautomatically and excluded from further analysis. The data were downsampled and subject to a high-pass filter $(>1 \mathrm{~Hz})$, as well as removal of powerline noise at $50 \mathrm{~Hz}$ using the CleanLine toolbox (Mullen, 2012). Cardioballistic artifacts were corrected via weighted average artifact subtraction (Goldman et al., 2000). Time-frequency transformation was conducted in the Fieldtrip toolbox (Oostenveld et al., 2011). A sliding window multitaper method (length, $4 \mathrm{~s}$; step size, $0.05 \mathrm{~s}$; spectral smoothing, $\pm 1 \mathrm{~Hz}$; Slepian tapers, 7) was applied to the preprocessed time series, resulting in spectrograms and cross-spectrograms from 1 to $100 \mathrm{~Hz}$ in steps of $0.25 \mathrm{~Hz}$. Spectrograms were segmented into $10 \mathrm{~s}$ nonoverlapping epochs and averaged across epochs. Power spectra were calculated by taking median values of the averaged spectrogram across time. To reduce intersubject variability, relative power was obtained by dividing the absolute power by the total power across the spectrum. The relative spectral power was categorized into six canonical frequency bands (Snyder et al., 2015), namely, delta $(1-4 \mathrm{~Hz})$, theta $(4-8 \mathrm{~Hz})$, alpha $(8-12 \mathrm{~Hz})$, beta $(12-30 \mathrm{~Hz})$, low gamma $(30-60 \mathrm{~Hz})$, and high gamma $(60-100 \mathrm{~Hz})$. The strength of neural synchronization between two recording sites was estimated from the cross-spectrogram. To minimize spurious synchrony between channels, we analyzed phase-lagged synchronization using debiased weighted phase-lag index (dwPLI) to confine analysis to noninstantaneous correlations, which has been demonstrated to be robust against volume conduction, noise, and sample-size bias (Vinck et al., 2011). For each EEG dataset, a $21 \times 21$ matrix of dwPLI values was generated for each frequency bin. Band-specific synchronization strength was calculated by averaging dwPLI values across frequency bins within the band.

\section{MRI data analysis}

The preprocessing of monkey data was implemented in the SPM 8.0 toolbox (http://www.fil.ion.ucl.ac.uk/spm) and the FMRIB Software Library toolbox (FSL; https://fsl.fmrib.ox.ac.uk/fsl/fslwiki). First, the initial 10 volumes of imaging data were discarded. Then the field map images of each monkey were applied to compensate for the geometric distortion of EPI images using FSL FUGUE. After slice-timing correction and motion correction, the corrected images were normalized to the standard space of the monkey F99 atlas (Van Essen et al., 2012) using an optimum 12-parameter affine transformation and nonlinear deformations, and then resampled to $2 \times 2 \times 2 \mathrm{~mm}$ voxels and spatially smoothed with a $4 \mathrm{~mm}$ FWHM isotropic Gaussian kernel. Six head motion parameters, and ventricle and white matter signals were removed from the smoothed volumes using linear regression. Linear drift of the volumes was removed, and a temporal filter $(0.01-0.1 \mathrm{~Hz})$ was performed. On the other hand, the preprocessing of human data was performed by the Preprocessed Connectomes Project (PCP; http:// preprocessed-connectomes-project.org/abide) using the Data Processing Assistant for Resting-State fMRI (DPARSF) Toolbox (Yan and Zang, 2010). Preprocessing steps included slice-timing correction, motion correction, spatial normalization into MNI space, reslicing to $3 \times 3 \times 3 \mathrm{~mm}$ voxels, and smoothing with a Gaussian kernel (FWHM, $6 \mathrm{~mm}$ ). Friston24 parameters of head motion, and white matter and ventricle signals were regressed out, followed by linear drift correction and temporal filtering $(0.01-0.1 \mathrm{~Hz})$. For more details, readers may refer to the description in the PCP (http://preprocessed-connectomes-project.org/abide/ dparsf.html).

\section{Network construction of monkey and human brains}

The cortical organization of both monkeys and humans was parcellated mainly based on the Regional Map template (Kötter and Wanke, 2005), with addition of subcortical parcellation from the INIA19 (Rohlfing et al., 2012) and Freesurfer templates (Fischl et al., 2002). This generated a whole-brain template with a total of 94 regions of interest for both monkeys and humans (Lv et al., 2016). Pearson's correlation coefficients between the mean time courses of any pair of regions were calculated to represent their functional connectivity, resulting in a $94 \times 94$ connectivity network matrix. Fisher's $z$-transformation was then applied to the connectivity matrix, which was subject to a covariate-regression procedure before group comparison. For the monkey data, age, age squared, and sex were regressed out of connectivity matrices using a general linear model. For the human data, FIQ and scanning site were also regressed out of connectivity matrices. 


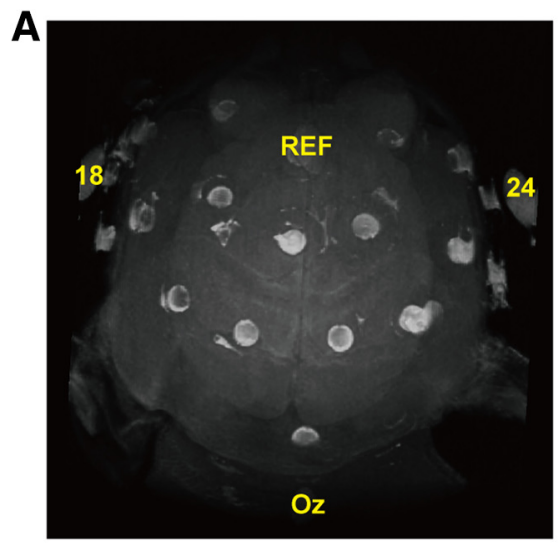

C

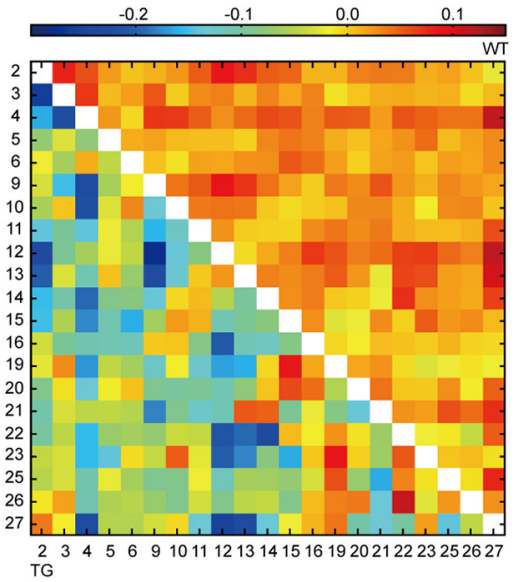

B

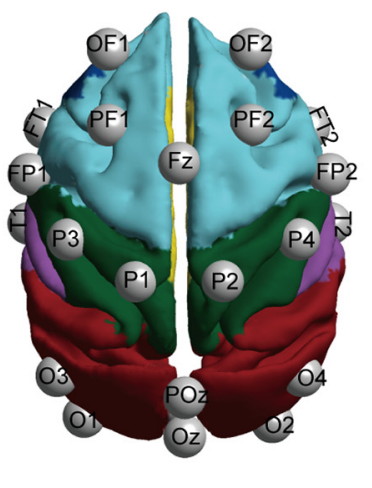

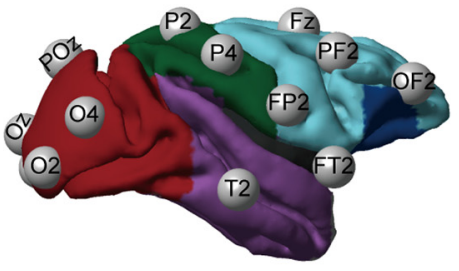

Orbitofrontal

Prefrontal

Parietal

Occipital

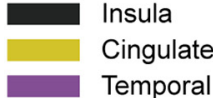

D

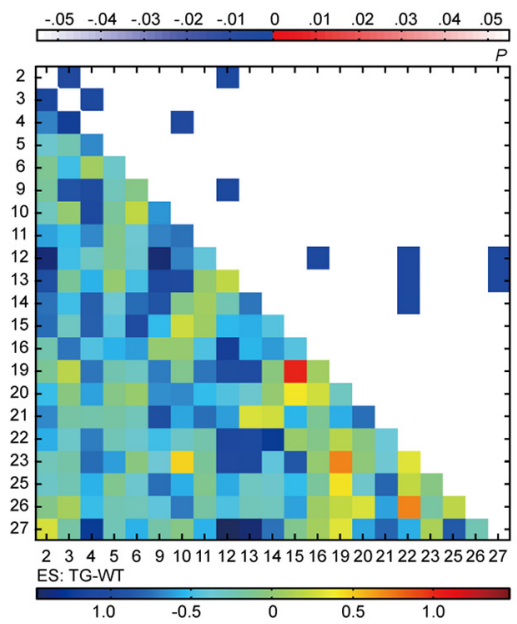

E
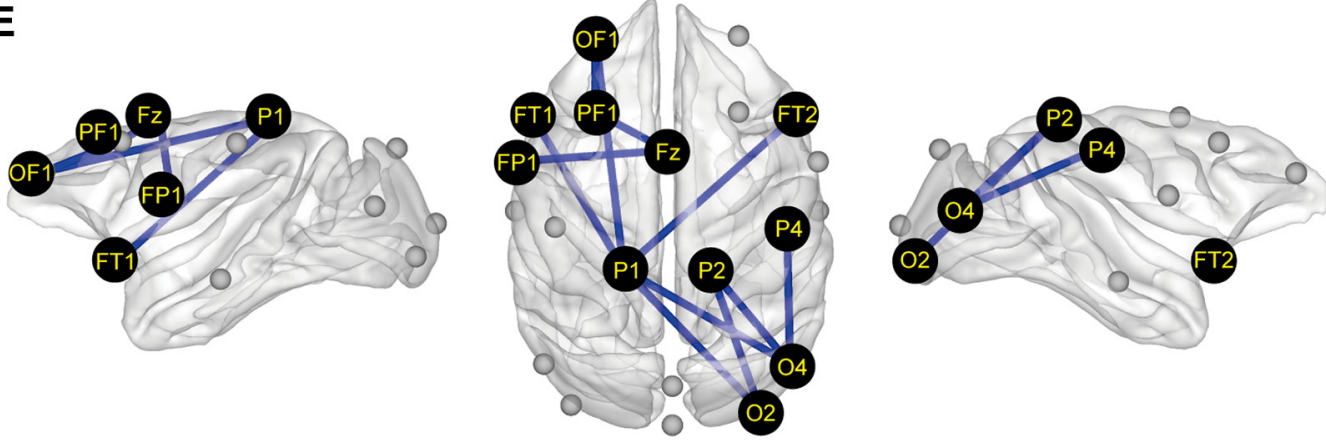

F

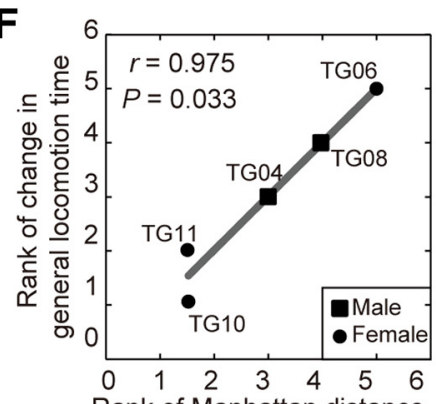

G

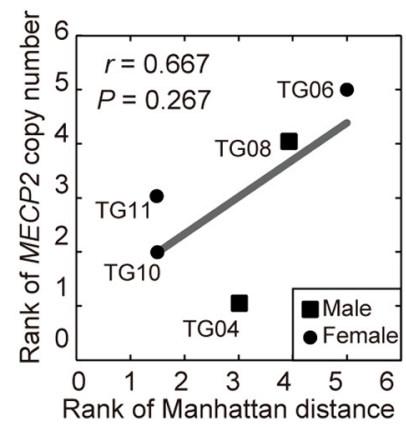

Figure 3. Decreased $\beta(12-30 \mathrm{~Hz})$ synchronization in fronto-parieto-occipital networks in TG monkeys. $\boldsymbol{A}$, Three-dimensional reconstruction of monkey TG04 wearing the EEG cap based on T2-weighted MRI images. Bright circles indicate electrode rings. Bright ellipses above circles indicate marked electrodes, including the reference (REF), most posterior (0z), and most lateral (18/ 24) electrodes. B, Spatial organization of 21 active electrodes after mapping to the cortical surfaces of the $F 99$ template brain. The electrodes were named after spatial location. $\boldsymbol{C}$, Averaged covariate-free neural synchronization networks of TG (bottom left) and WT (top right) monkeys. The numbers indicate the electrode ID. The color bar denotes the logarithm of dwPLI. D, ESs (bottom left) and $p$ values (top right) of the statistical comparison between TG and WT groups. Eleven connections widely distributed in fronto-parieto-occipital networks were identified significantly decreased in TG monkeys (edgewise, $p<0.005$; cluster-level corrected, $p=0.010$ ). $\boldsymbol{E}$, Topographic distribution of abnormal connections was illustrated in the F99 template brain. $\boldsymbol{F}, \boldsymbol{G}$, 
Subject stratification based on functional connectivity patterns

We used data-driven stratification of functional connectivity to reveal clusters or subgroups in ABIDE cohorts. A total of 230 human samples (90 autism subjects and 140 TDCs) were used to generate a $230 \times 230$ similarity (spatial correlation) matrix by calculating Pearson's correlation coefficient between the brain networks (lower triangle of network matrix) of any two participants. This interindividual similarity matrix was rarefied as the maximum threshold was determined (Pearson's $r=0.55$; sparsity $=0.42$ ) to keep the whole matrix connected. A community detection method was then applied to stratify human participants based on the interindividual similarity of brain connectivity networks using the Brain Connectivity Toolbox (Rubinov and Sporns, 2010). Two clusters or subgroups were detected automatically by maximizing the modularity of the similarity matrix. For the entire cohort and the two subgroups, network homogeneity within autism subjects and TDCs was evaluated by averaging the network similarities between each participant and all other participants in the group.

\section{Experimental design and statistical analysis}

Genetic analysis

Functional enrichment analysis. After identification of gene modules in WGCNA, we performed enrichment analysis on MECP2 coexpressed genes. The significance of the enrichment analysis was corrected with Benjamini-Hochberg false discovery rate (FDR; $p<0.01$ ).

Transcriptome analysis. The expression level of MECP2 coexpressed genes revealed in WGCNA were statistically compared between three MECP2-overexpressed and four WT monkeys via independent twosample $t$ tests. Uncorrected $p$ values $<0.025$ were considered significant.

\section{Behavioral analysis}

Locomotive behavior in home cage and peer separation test. Repetitive index and general locomotion time were analyzed in TG and WT groups. Group differences in the two measures were statistically tested using independent two-sample $t$ tests.

Reversal learning task. Perseverative and regressive error rates in the reversal learning task were statistically compared between two groups via independent two-sample $t$ tests. For each session with 80 trials, the error rates were calculated based on every 10 trials performed after rule switch.

\section{Monkey EEG}

Relative power of 21 recording sites and pairwise neural synchronization were obtained from 5 TG and 16 WT monkeys for six frequency bands. Group comparisons of power and synchronization were conducted separately for individual frequency bands. Covariates including age, age squared, and sex were controlled in a linear regression model before group comparison. Group differences in relative power were tested using independent two-sample $t$ tests, with Bonferroni correction for multiple comparisons. Effect size was estimated for each channel via Hedges' $g$ value.

To further localize specific pairs of brain regions in which neural synchronization was altered by MECP2 overexpression, the network-based statistical approach was used to seek out between-group differences (Zalesky et al., 2010). This approach copes with the multiple-comparison problems of comparing connectivity data with $N$ nodes and $N \times(N-1) / 2$ connections or edges by evaluating the null hypothesis at the level of connected subnetworks rather than individual connections. Specifically, an independent two-sample $t$ test was applied to each connection in the covariates-free dwPLI matrix. A primary threshold $(p<0.005)$ was applied to the edge-level statistical map, which may result in one or more connected clusters or subnetworks. A nonparametric permutation approach (10,000 times) was then used to ascribe a cluster-level $p$ value to each subnetwork based on its size. Subnetworks with

$\leftarrow$

Association of the overall abnormality in $\beta$-synchronization (Manhattan distance) with general locomotion time change after peer separation $(\boldsymbol{F})$ and MECP2 copy number (G). Associations were evaluated via Spearman's rank correlation for TG monkeys only. a cluster-level $p<0.05$ were reported. Effect sizes were estimated for all connections via Hedges' $g$ value.

\section{Monkey and human $f M R I$}

A connectivity network between 94 brain regions was constructed for each monkey and human subject. Group comparison on the connectivity network was conducted for monkeys (5 TG vs $11 \mathrm{WT}$ ) and human subgroups ( 49 subjects with autism vs 72 TDCs for subgroup 1; 41 subjects with autism vs 68 TDCs for subgroup 2; for details, see Results), respectively. The same network-based statistic as described in EEG analysis was applied with edgewise threshold of the significance level set at $p=0.001$ and cluster-level correction of $p<0.05$ to adjust the multiple comparison. Effect size (ES) of all connections were measured by Hedges' $g$ value. The ES of each brain region $(E S n)$ was calculated by summing up the ESs of all edges with significant group difference connecting to this region. The ESn values were divided by the absolute maximum ESn, leading to a scale between -1 and 1 .

To assess the distribution of these disrupted edges within and between lobes, we used the standardized residuals (Sheskin, 2003) to adjust the bias caused by the Regional Map parcellation in which the number of nodes is not equal in different lobes. The observed edge distribution was compared with expected distribution where all edges are randomly distributed across lobes. The significance of observed distribution was estimated via a nonparametric statistical test and adjusted for multiple comparisons via Bonferroni correction.

We further calculated the Spearman's correlation coefficient between connectivity strength of group-different edges and behavioral measures or autistic symptoms to derive the corresponding abnormal subnetworks. For the monkey data, edgewise threshold of the significance level was set at $p=0.01$ for spontaneous locomotion in home cage (data available for 16 monkeys) and $p=0.05$ for behavioral measures from peer separation test and reversal learning task (data available for 8 and 9 monkeys, respectively). For the human data, edgewise threshold was set at $p=0.01$ (data available for 29 and 25 patients in subgroup 1 and subgroup 2, respectively). Cluster-level correction of $p<0.05$ was applied to adjust for multiple comparisons via network-based statistics.

\section{Gene-circuit behavior association}

We adopted Manhattan distance (MD) to quantitatively describe the overall abnormal extent of the connectivity fingerprint of individual TG monkeys or autism subjects relative to controls (Neubert et al., 2014; Mars et al., 2016). Such a "distance measure" can determine whether different fingerprints are "close" or "far" from each other. For neural connectivity analysis on EEG data, MD based on dwPLI of edges with abnormal neural synchronization was calculated in each frequency band for each TG monkey. For functional connectivity analysis on fMRI data, MD based on the connectivity strength of edges within the abnormal neural network or subnetworks were calculated for each TG monkey and each autism subject. The significance of MD was estimated via a permutation test where group labels were randomly shuffled 10,000 times. Spearman's correlation analysis was performed in a pairwise manner to probe associations among gene (MECP2 copy number), circuits (MD based on EEG or fMRI), and behaviors (home cage, peer separation test, and reversal learning task) in five TG monkeys. As no genetic information was available for the human data, circuit-behavior associations were calculated in autism between MD based on the abnormal fMRI networks and clinical scores of Autism Diagnostic Observation Schedule (ADOS).

\section{Cross-species comparison of dysconnectivity fingerprints}

Group-level comparison within each species revealed disrupted brain connections and regions in TG monkeys and autistic patients. We examined the cross-species overlap of disrupted brain connections and regions, and statistically tested the cross-species similarity in brain disruption by comparing subject-specific dysconnectivity fingerprints via MD. The dysconnectivity fingerprint was defined as the effect sizes of abnormal brain regions shared in both species. We defined the 
fingerprint based on characteristics of brain regions rather than connections as the former is more likely to be evolutionarily conserved (van den Heuvel et al., 2016; Mars et al., 2018) and reflects abnormality of a wide range of connections including subject-specific abnormal connections (Mars et al., 2016). A subject-specific fingerprint was obtained by comparing the connectivity matrix of each "abnormal" subject with those of control subjects and summing up the edgewise effect size (Hedge's $g$ ) for each abnormal region shared between two species.

MD between each two "abnormal" subjects was calculated based on their dysconnectivity fingerprints. The null hypothesis to reject was that distances between transgenic monkeys and human patients $(\mathrm{M}-\mathrm{H})$ were statistically equal to those between two human patients $(\mathrm{H}-\mathrm{H})$. A nonparametric permutation test with 5000 permutations was used to test the hypothesis. M-H distances based on each TG monkey were compared with all $\mathrm{H}-\mathrm{H}$ distances individually and subject to Bonferroni correction for multiple comparisons.

Data availability

Data are available from Zheng Wang on reasonable request.

\section{Results}

\section{Association of $M E C P 2$ coexpression network with GABA function}

We found that MECP2 belonged to a module consisting of 159 coregulated genes and was positively correlated with the $\mathrm{ME}$ (Pearson's $r=0.66, p<2.2 \times 10^{-16}$; Fig. $1 B, C$ ). Further functional enrichment analysis revealed that this module was highly associated $(p<0.01$, FDR corrected) with mood disorders listed in the DisGeNET database, four gene pathways listed in the NCBI BioSystem database including GABA function, and three cellular components listed in the Gene Ontology database including clathrin-sculpted GABA vesicular transport (Fig. 1D).

By comparing the expression abundance of MECP2 toplinked genes between TG and WT monkeys, we found that most of the genes that exhibit the strongest association with MECP2 expression were dramatically regulated in TG monkeys. Among those genes, 5 were significantly upregulated (Fig. 1C, pink color), while 18 were significantly downregulated (Fig. $1 C$, purple color). More details of these dysregulated genes are provided in Figure $1 E$.

\section{Behavioral deficits in TG monkeys}

The inter-rater reliability of repetitive circular and general locomotion time was $96.3 \%$ and $98.1 \%$, respectively. The total time spent in general locomotion for TG monkeys was significantly greater than for WT monkeys $\left(t\right.$ test: $t_{(19)}=3.14, p=0.005$, Hedge's $g=1.55)$. The repetitive index of locomotion was also significantly higher in TG monkeys $\left(t\right.$ test: $t_{(19)}=3.94, p=0.001$, Hedge's $g=1.94$ ), as presented in Figure $2 B$.

Both TG and WT monkeys showed increased locomotion time after peer separation compared with the adaptation period $\left(t\right.$ test: $t_{(4)}=-4.14, p=0.014 ; t$ test: $t_{(6)}=-2.61, p=0.040$, respectively). No group difference was found in the amount of change in locomotion time ( $t$ test: $t_{(10)}=1.61, p=0.138$, Hedge's $g=0.87$; Fig. $2 C$, left). TG monkeys also showed a marginally significant increase in repetitive index ( $t$ test: $t_{(4)}=-2.66, p=0.056$ ), whereas WT monkeys showed no such change $\left(t\right.$ test: $t_{(6)}=-0.64$, $p=0.547)$. There was a significant group difference in the amount of change in repetitive index ( $t$ test: $t_{(10)}=2.48, p=0.032$, Hedge's $g=1.34$; Fig. $2 C$, right). No group difference in induced behavioral changes was found in the case of unfamiliar peer separation in either general locomotion time ( $t$ test: $t_{(10)}=-0.48, p=0.643$ ) or repetitive index ( $t$ test: $\left.t_{(10)}=0.06, p=0.953\right)$.
TG and WT monkeys spent an equivalent time to acquire both stimulus-directed touching $\left(t\right.$ test: $\left.t_{(7)}=1.11, p=0.303\right)$ and stimulus-reward association rules $\left(t\right.$ test: $\left.t_{(7)}=-0.89, p=0.401\right)$, and omitted a similar number of trials ( $t$ test: $t_{(7)}=-0.39$, $p=0.710)$ during the reversal learning task. Notably, TG monkeys exhibited a significantly greater regressive error rate for the first 20 performed trials than WT monkeys (Fig. 2F, G; $t$ test: $t_{(7)}=2.56, p=0.038$, Hedge's $g=1.47$ ), while they did not demonstrate an increased rate of perseverative errors ( $t$ test: $t_{(7)}=$ $0.81, p=0.443)$.

\section{Decreased $\beta$-synchronization in TG monkeys}

Group difference was found to be significant in the $\beta$ band at the network level, as shown in Figure $3 C$, together with the corresponding ES (Hedges' $g$ value) of individual connections (Fig. $3 D)$. We identified a fronto-parieto-occipital network that showed a statistically significant decrease in $\beta$-synchronization in TG monkeys (Fig. 3E; network-based statistic: edgewise, $p<0.005$; cluster-level corrected, $p=0.010$ ). This network consisted of two connections within the frontal lobe (OF1-PF1, and $\mathrm{PF} 1-\mathrm{Fz}$ ) and four fronto-parietal connections (Fz-FP1, OF1-P1, FT1-P1, and FT2-P1) in the left hemisphere, and five parietooccipital connections $\left(\mathrm{P} 1-\mathrm{O}_{2}, \mathrm{P} 1-\mathrm{O} 4, \mathrm{P} 2-\mathrm{O}_{2}, \mathrm{P} 2-\mathrm{O} 4\right.$, and $\left.\mathrm{P} 4-\mathrm{O} 4\right)$ in the right hemisphere. The overall abnormality in $\beta$-synchronization was significantly correlated with locomotion time change after familiar peer separation (Spearman's $r=0.975$, $p=0.033$; Fig. $3 F$ ), but not with the MECP2 copy number (Spearman's $r=0.667, p=0.267$; Fig. $3 G$ ). No significant group difference in neural synchronization of other frequency bands was observed. No significant group difference in the relative power of either frequency band was observed after correction for multiple comparisons.

\section{Aberrant functional connectivity in TG monkeys}

Group-averaged connectivity networks of TG and WT monkeys are presented in Figure $4 A$. The ES of each connectivity is presented in the bottom left triangle in Figure $4 B$. We identified a total of 50 functional connections that showed significant differences between TG and WT groups (network-based statistic: edgewise, $p<0.001$; cluster-level corrected, $p=0.019$; Fig. $4 B$, top right triangle). Of 94 nodes, 40 regions were found containing abnormal connections with remarkably large effect sizes. The degree of abnormality of each node [i.e., the sum of effect sizes of all abnormal edges connecting to this node $(E S n)]$ is presented in the interlayer of Figure $4 C$. The right primary somatosensory cortex (S1) showed decreased connectivity with a large ESn (top 25\%; Fig. 4C, blue bars). In contrast, increased connectivity regions with the top 25\% largest ESn (red bars) covered a wide range of frontal cortex including left polar cortex (PFCpol), right centrolateral prefrontal cortex (PFCcl), right lateral orbitofrontal cortex (PFCol), and frontal eye field, cingulate areas including bilateral retrosplenial cortex cingulate $(\mathrm{CCr})$ and left anterior cingulate cortex, and other areas including right medial parietal cortex and left parahippocampus cortex (PHC). The distribution of altered functional connections in brain lobes are summarized in Figure $4 D$ ( $z$-scores in bottom left triangle) after the nonuniform parcellation of the brain template was accounted for. The distribution was preferentially biased to prefrontal and cingulate cortices (permutation test, $p<0.05$, Bonferroni correction; Fig. $4 D$; top right triangle).

Fourteen edges that mostly stemmed from left PFCpol and left PHC were correlated with the regressive error rate in the reversal learning task (network-based statistic: edgewise, $p<0.05$; 
A

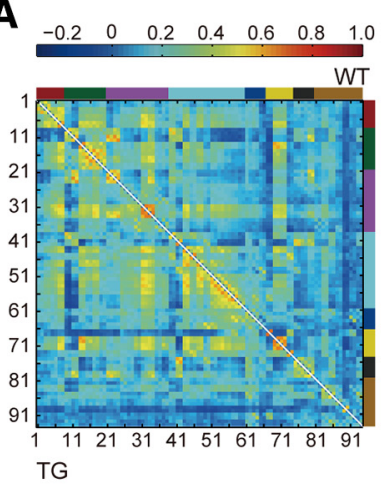

B

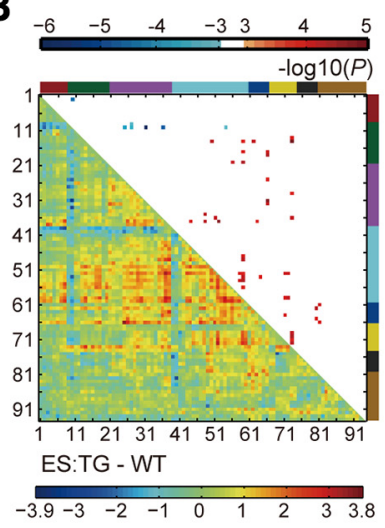

D

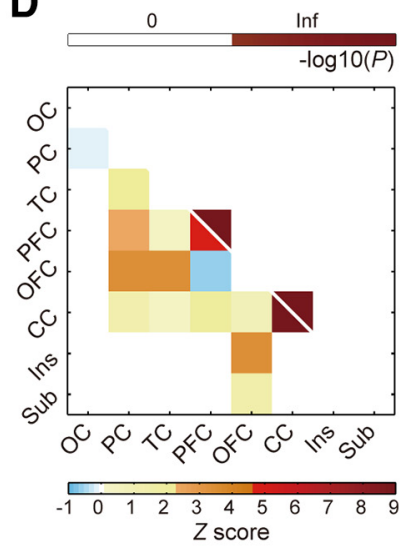

C
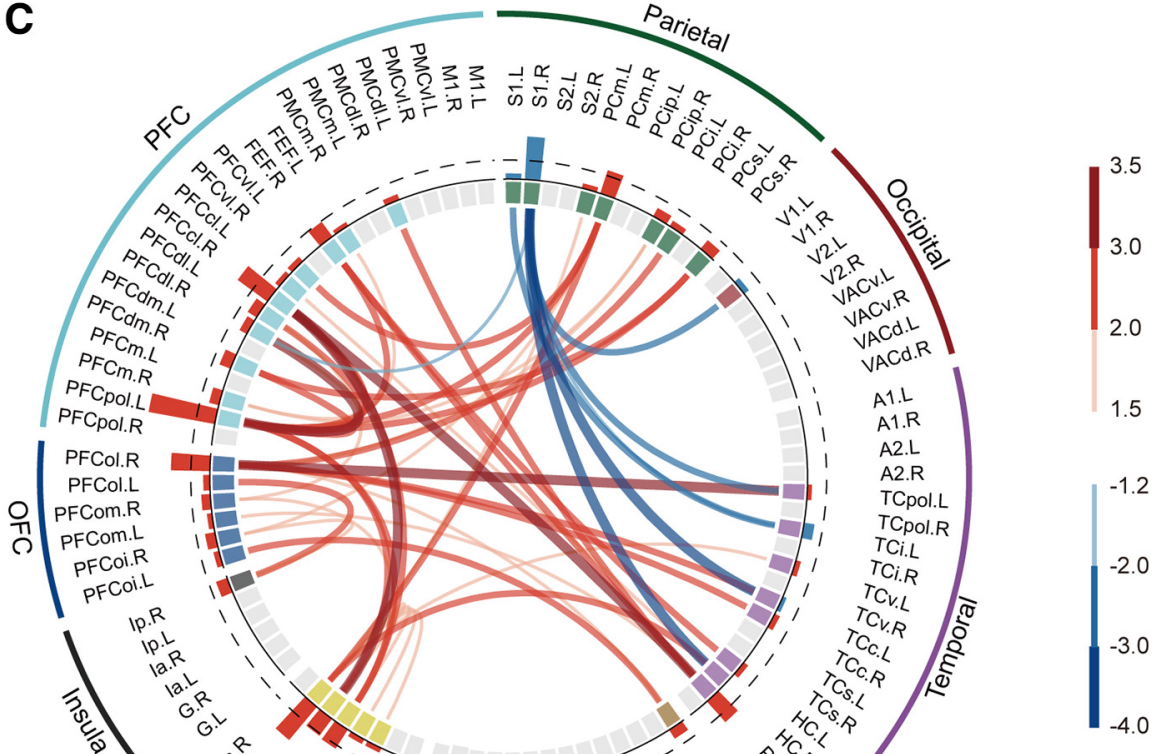

ES: TG - WT

E

Regressive error rate

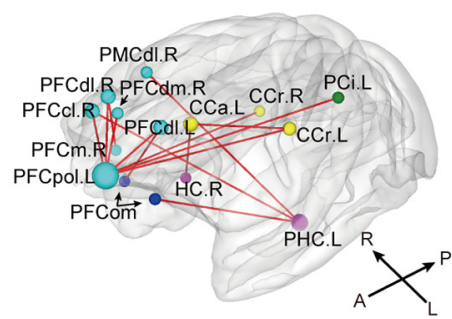

F

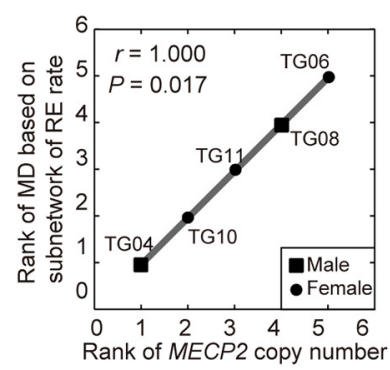

G

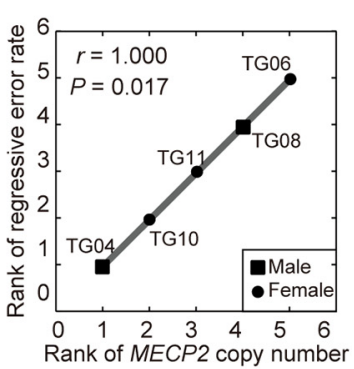

Figure 4. Disrupted neural circuits in transgenic monkeys. $A$, Covariate-free connectivity network matrices for TG (bottom-left) and WT (top-right) monkeys. $B$, ESs of TG versus WT (bottomleft) are shown with corresponding $p$ values (top-right; cluster-level corrected, $p<0.05$; edgewise, $p<0.001$ ). Brain nodes are sorted and organized according to the regions/lobes. $C$, Disrupted functional connections and brain nodes in TG monkeys. Red and blue bars in the interlayer indicate positive and negative ESn values, respectively. The dashed line labels the top 25\% of absolute ESn values. $\boldsymbol{D}$, Spatial distribution of disrupted connections across the brain (bottom left) and corresponding statistical significances (top right; $p<0.05$, Bonferroni correction). Inf, Infinite; $O C$, occipital cortex; $P$ C, parietal cortex; $C$, cingulate cortex; Ins, insula; Sub, subcortical areas. $E$, Subnetworks associated with regressive error rates in the reversal learning task $(p<0.05$, corrected). $\boldsymbol{F}$, Gene-circuit association between MECP2 copy number and circuit abnormality. RE, Regressive error. G, Gene-behavior association between MECP2 copy number and regressive error behavior. Associations were evaluated via Spearman's rank correlation for TG monkeys only.

cluster-level corrected, $p=0.006$; Fig. $4 E$ ). Strikingly, the $M E C P 2$ copy number in TG monkeys was significantly associated with the MD of these dysfunctional connections (Spearman's $r=1.000, p=0.017$; Fig. $4 F)$ and with the regressive error rate
(Spearman's $r=1.000, p=0.017$; Fig. $4 G$ ). Seven edges that mostly emanated from right $S 1$ were correlated with general locomotion time in home cage (network-based statistic: edgewise, $p<0.01$; cluster-level corrected, $p=0.005$ and $p=0.037$, 
A

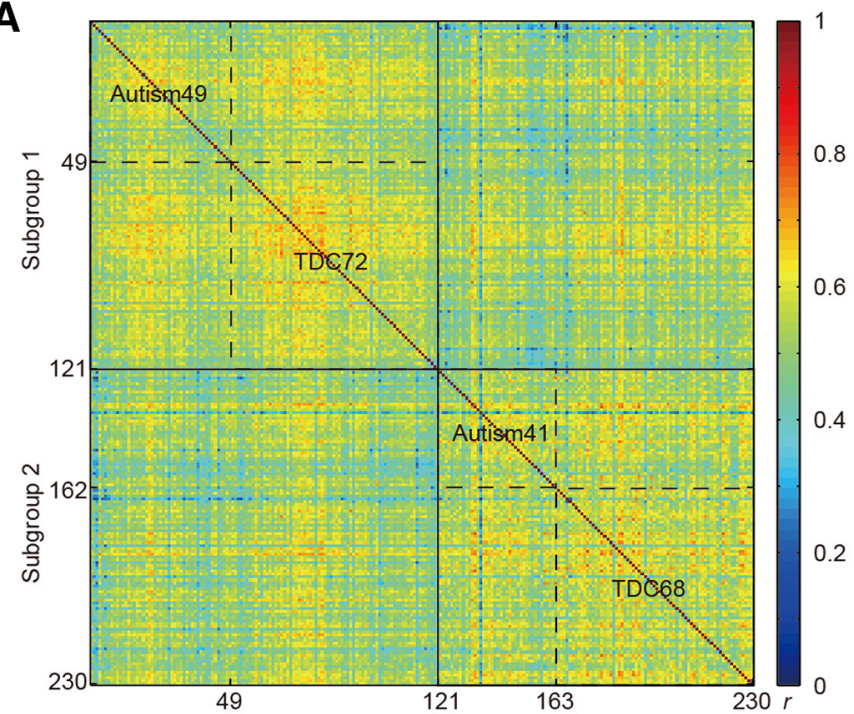

B

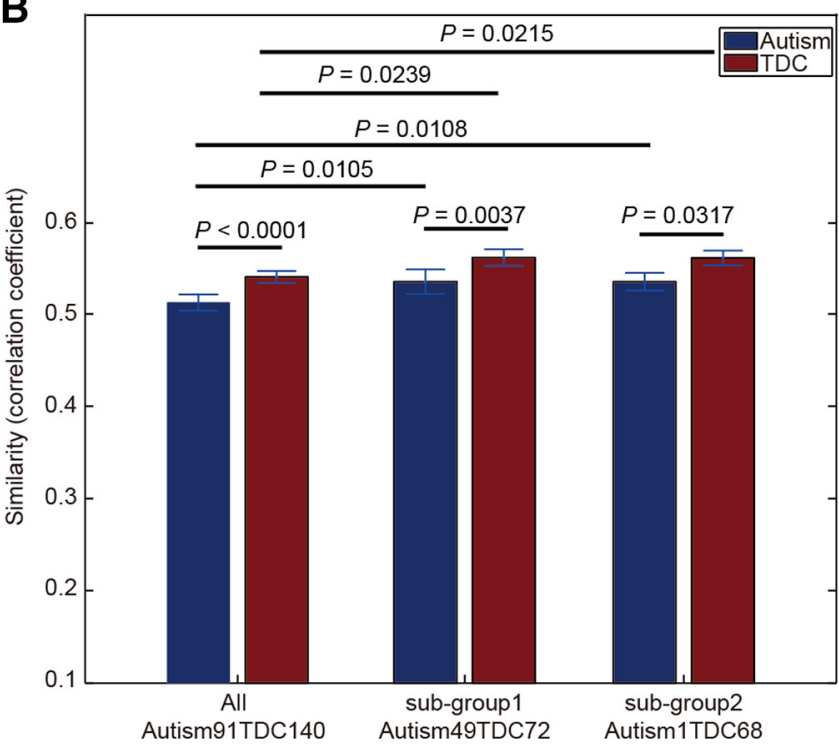

C
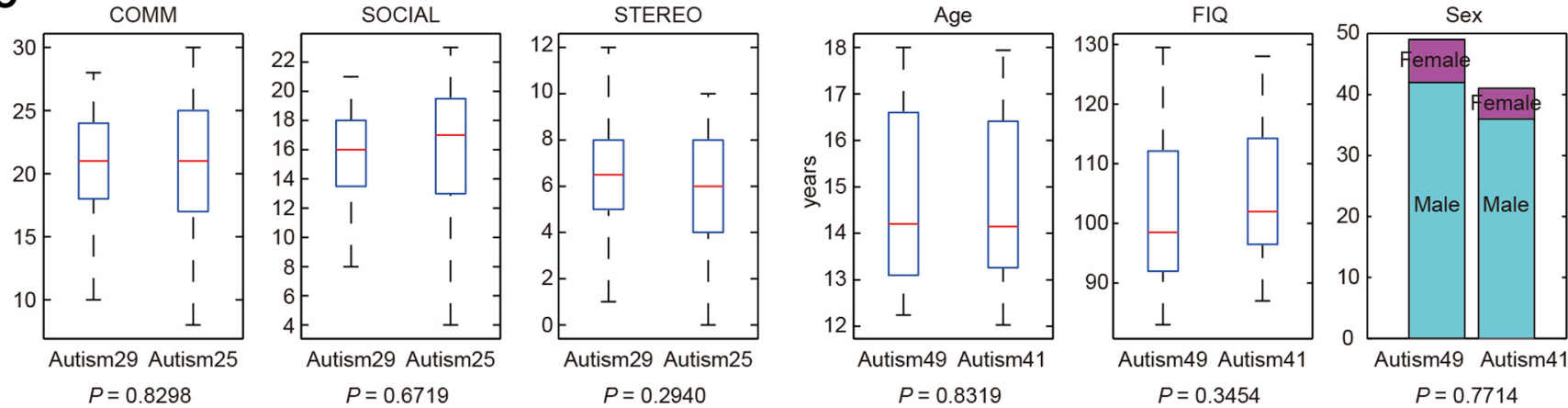

Figure 5. Subject stratification based on functional connectivity patterns. $A$, An interindividual network similarity matrix of 230 participants clustered by a community detection algorithm. Two subgroups or clusters were derived out of this cohort, one with 49 individuals with autism and 72 TDCs (subgroup 1), and another with 41 individuals with autism and 68 TDCs (subgroup 2). Each row represents the similarities between the brain network of a single subject and that of all other subjects. Color bar, Pearson's correlation coefficients ( $r$ ). $\boldsymbol{B}$, Group comparison of interindividual network similarity. Brain network similarities of TDCs are always significantly higher than those of autism for both the entire cohort and two subgroups $(10,000$ times of permutation test). Brain network similarities of subgroups are significantly higher than those of the autism and TDC groups, respectively (10,000 times of permutation test). Error bars, SD. C, Comparisons of demographic information and clinical scores of human autism between subgroup 1 and subgroup 2. Note that clinical scores are only available for some patients in both subgroups ( 29 and 25 for subgroup 1 and 2, respectively). In the boxplot, the central mark is the median, and the edges of the box are the 25th and 75th percentiles. The whiskers extend to the most extreme data points. COMM, Communication total subscore of the classic ADOS; SOCIAL, social total score of the classic ADOS; STERE0, stereotypic behaviors and restricted interests total subscore of the classic ADOS.

respectively). However, the overall abnormality in this subnetwork was not associated with $M E C P 2$ copy number (Spearman's $r=0.500, p=0.450$ ).

\section{Aberrant functional connectivity in subgroups of autistic patients}

Two clusters were derived from the clinical cohort based on the functional connectivity patterns, as follows: subgroup 1 consisted of 49 subjects with autism (age, $14.79 \pm 1.78$ years; 7 females) and 72 TDCs (age, $14.65 \pm 1.65$ years; 16 females), and subgroup 2 consisted of 41 subjects with autism (age, $14.71 \pm 1.82$ years; 5 females) and 68 TDCs (age, $14.71 \pm 1.73$ years; 15 females; Fig. $5 A$ ). No statistical differences in phenotypic and demographic characteristics were found between two subgroups (Fig. 5C). No statistical differences in the signal-to-noise ratio of the imaging data were found between two subgroups ( $t$ test: $t_{(228)}=0.87, p=0.384$ ).

As in the monkey dataset, we repeated the same statistical analysis for both subgroups in parallel. In subgroup 1, 32 significant hypoconnections were observed in 27 brain nodes (network-based statistic: edgewise, $p<0.001$; cluster-level corrected, $p=0.026$; Fig.
$6 B, C)$, most of which were located between temporal cortex (TC) and PFC, between TC and orbitofrontal cortex (OFC), between TC and occipital cortex, and within TC ( $p<0.05$, Bonferroni correction; Fig. $6 D$ ). Regions with the top $25 \%$ largest $E S n$ values were right PFCpol, right PFCol, bilateral ventral TC (TCv), right TC polar (TCpol), right superior TC, and right primary auditory cortex (A1; Fig. 6C, blue bars of interlayer). In contrast, no abnormal connections in subgroup 2 withstood correction for multiple comparisons. In 29 of 49 participants with research-reliable ADOS scores, we observed a significant correlation between communication scores and their MD (Spearman's $r=0.416, p=0.025$ ), but not with social interaction or restricted, repetitive behavior (Fig. 6E). No subnetworks underlying specific symptoms were further derived from the disrupted network.

\section{Cross-species comparison of dysconnectivity fingerprints}

Although no abnormal connections were shared between TG monkeys and patients with autism in subgroup 1 (Figs. $4 C$, $6 C)$, there were overlaps in abnormal brain nodes including lateral prefrontal areas (left $\mathrm{PFCcl}$, right $\mathrm{PFCvl}$ ), lateral 
A

$\begin{array}{rrrrrrr}-0.2 & 0 & 0.2 & 0.4 & 0.6 & 0.8 & 1.0\end{array}$

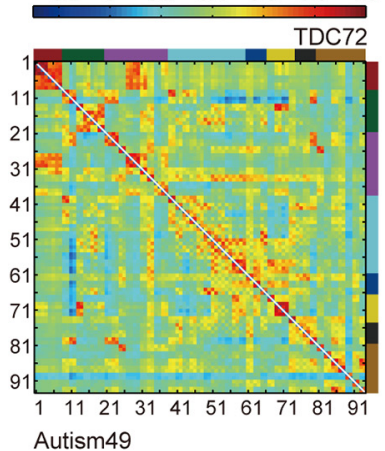

B

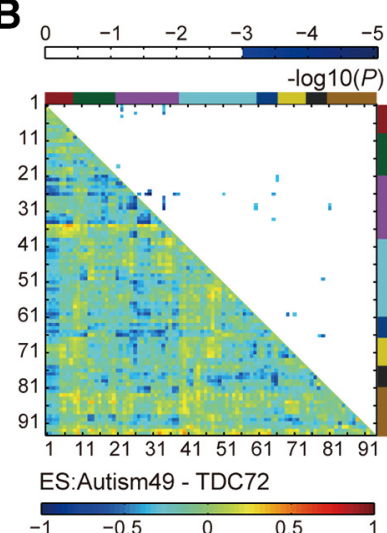

D

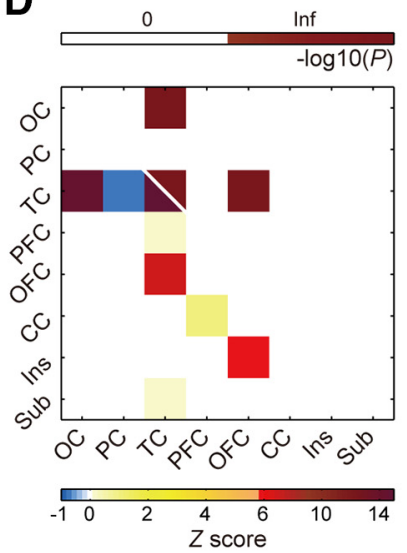

C

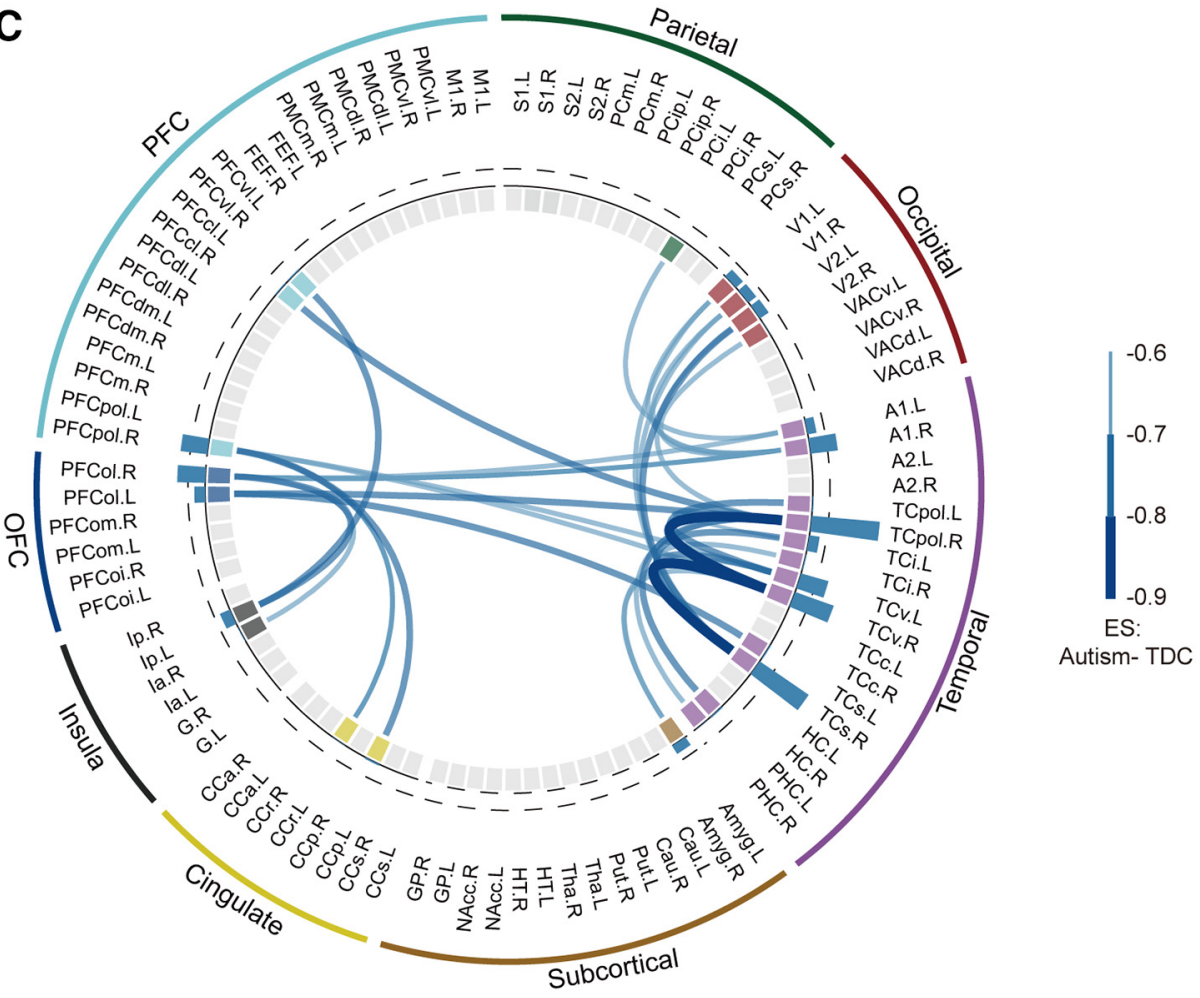

E
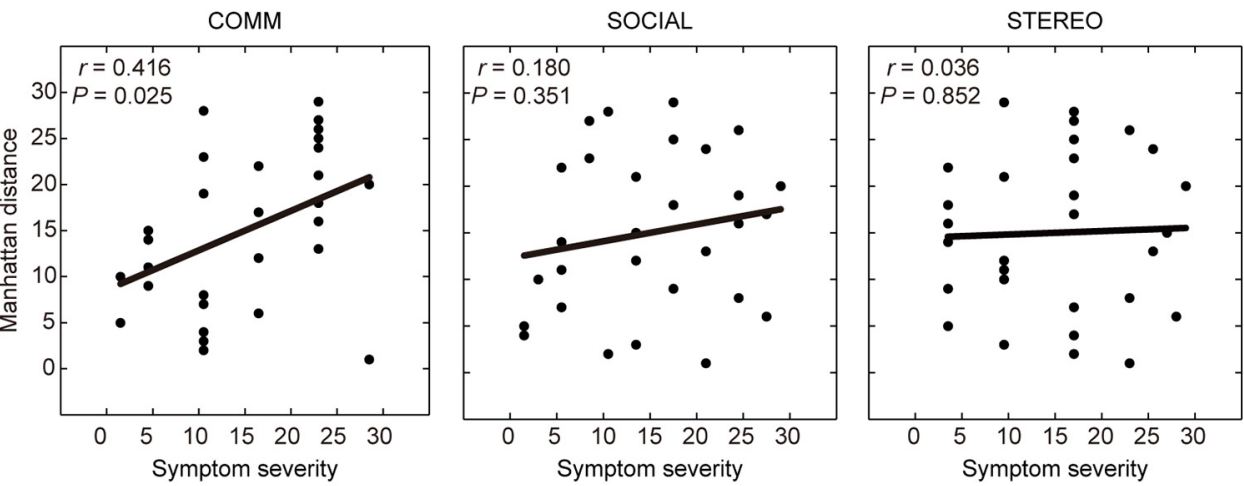

Figure 6. Disrupted neural circuits in a subgroup of patients with autism. $A$, Covariate-free connectivity network matrices for subjects with autism (bottom left) and TDC (top right) in subgroup 1. $\boldsymbol{B}$, ESs of autism versus TDC (bottom left) are shown with corresponding $p$ values (top right; cluster level, $p<0.05$; edgewise, $p<0.001$ ). The brain nodes are sorted and organized according to the regions/lobes. $C$, Disrupted functional connections and corresponding brain nodes in patients with autism compared with TDC. The blue bar in the interlayer indicates negative ESn, which was defined as the sum of the ESs of all abnormal connections to this node. The dashed line labels the top $25 \%$ of absolute ESn. D, Spatial distribution of disrupted connections across the brain (bottom left) and the corresponding statistical significance (top right; $p<0.05$, Bonferroni correction). Inf, Infinite; OC, occipital cortex; PC, parietal cortex; $C C$, cingulate cortex; Ins, insula; Sub, subcortical areas. $\boldsymbol{E}$, Associations between Manhattan distances of each autism patient and the severity of dimensional symptoms in communication (left), social interaction (middle), and stereotyped behaviors (right), respectively. 

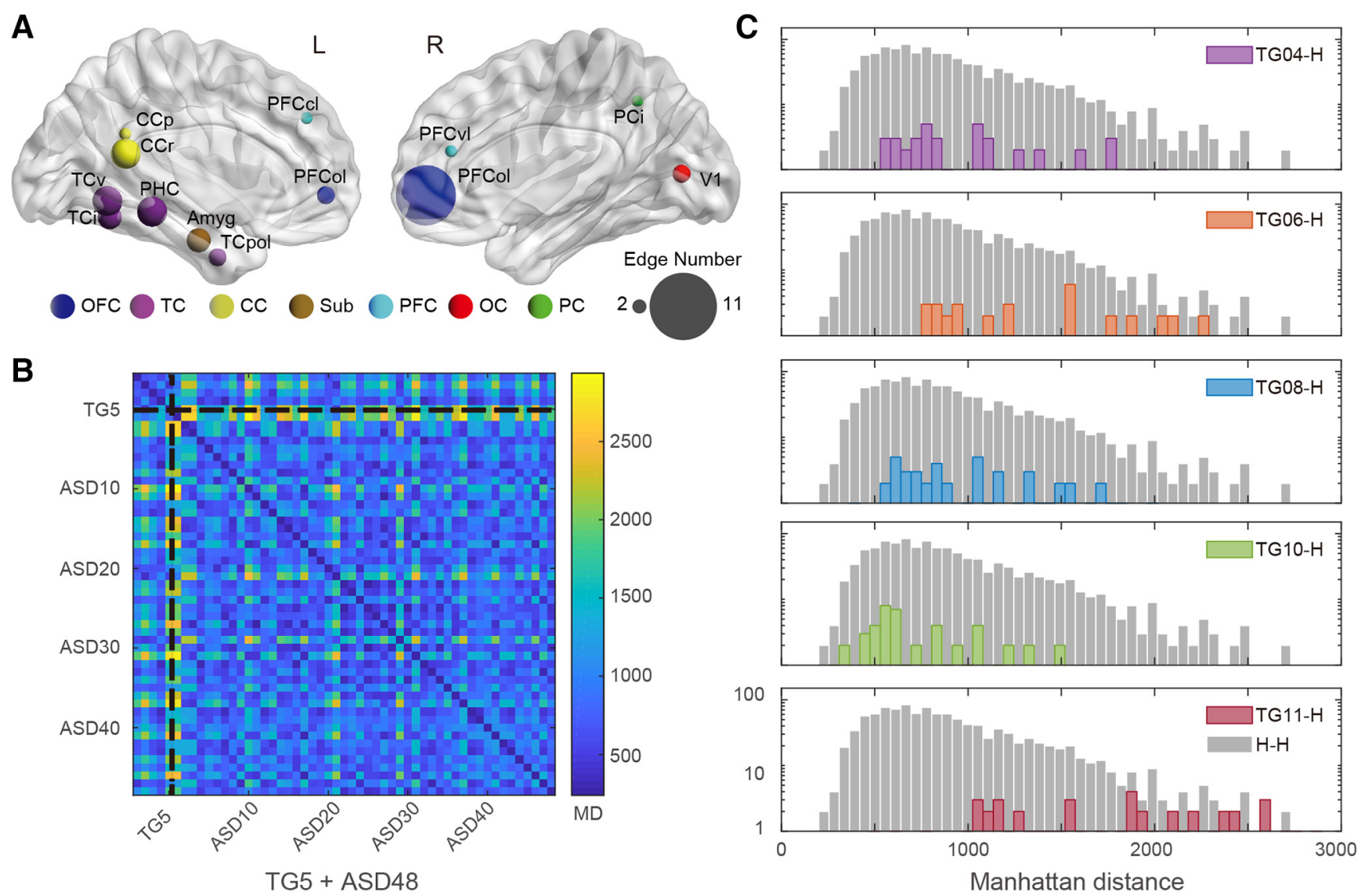

Figure 7. Cross-species comparison of dysconnectivity fingerprints in monkeys and humans. $\boldsymbol{A}$, Abnormal brain regions shared between transgenic monkeys and human patients. The size of nodes indicates the total number of abnormal edges connected to the node in both species. CC, Cingulate cortex; Sub, subcortical areas; OC, occipital cortex; PC, parietal cortex. B, MDs between subject-specific dysconnectivity fingerprints of the shared abnormal brain nodes. C, Distribution of cross-species distances between each transgenic monkey and autistic patients in subgroup 1 , compared with that between patients. Cross-species MD of TG04 $(p=0.225)$, TG08 $(p=0.250)$, and TG10 $(p=0.054)$ were not statistically different from within-patient MD, whereas TG11 $(p<0.001)$ and TG06 $(p<0.001)$ were. The $p$ values are obtained via permutation tests (5000 times) and subject to Bonferroni correction for multiple comparisons. The count frequency is indicated in a logarithmic $y$-axis. TG-H, distribution of MD between a specific transgenic monkey and each autistic patient in subgroup 1; H-H, distribution of MD between each two autistic patients in subgroup 1.

orbitofrontal areas (bilateral PFCol), left temporal areas (TCi, $\mathrm{TCV}$, and TCpol), left cingulate (posterior cingulate cortex and $\mathrm{CCr}$ ), left PHC, left amygdala, right inferior parietal area, and right primary visual area (Fig. 7A). MD between subject-specific dysconnectivity fingerprints of these shared brain nodes are presented in Figure $7 B$. One autism patient was excluded because the MDs between this patient and 23 (of 48) other patients were extremely large (3 SDs above the mean MD between two patients). The distribution of MDs between each TG monkey and all autism patients in subgroup1 were plotted against the distribution of MDs between each two autism patients (Fig. 7C). Cross-species MD of TG04 (permutation test, $p=0.225$ ), TG08 (permutation test, $p=0.250$ ), and TG10 (permutation test, $p=0.054$ ) were not statistically different from within-human MDs, indicating that the dysconnectivity profiles of these transgenic monkeys were comparable to those of autism patients. But the dysconnectivity profiles of TG11 (permutation test, $p<0.001$ ) and TG06 (permutation test, $p<0.001$ ) were different from those of autism patients.

\section{Discussion}

MECP2-related GABA dysfunction and $\beta$-desynchronization The frequency-dependent finding in electrophysiological activity likely reflects $M E C P 2$-induced dysfunction in specific molecular pathways. Evidence from transgenic rodents has demonstrated that $M E C P 2$ dysfunction alters synchrony and the overall excitation/inhibition balance in brain circuits, with greater influence on GABAergic neurons (Chao et al., 2010). The present transcriptome and functional enrichment analysis of macaque neocortex reveals a strong association between $M E C P 2$ coexpressed genes and GABA function, implying altered GABAergic action in the mutant monkeys. Moreover, results from both intracellular recording and genetic association studies suggest that generation of cortical $\beta$ oscillation depends on GABAergic neurotransmission (Whittington et al., 2000; Porjesz et al., 2002). As such, we propose that the disrupted $\beta$-synchronization observed in transgenic monkeys is very likely a consequence of the MECP2-induced malfunction of GABAergic neurons. Similar dysfunction of GABAergic signaling (Robertson et al., 2016) and reduction in $\beta$-synchronization during resting state (Shou et al., 2017) have been reported in ASD patients. Although no associations between spontaneous $\beta$-coherence and behavioral symptoms have been reported in human patients thus far, this may be a potential diagnostic marker for autism that merits future validation.

\section{Circuit and behavior disruptions in TG monkeys and autistic humans}

The prevailing hypothesis of disrupted cortical connectivity, in which deficiencies in the way the brain coordinates and 
synchronizes activity among different regions account for clinical symptoms of ASD, is central to the etiology and accurate diagnosis of ASD (Ecker et al., 2015; Hahamy et al., 2015; Kessler et al., 2016). However, human heterogeneity in terms of complex genetic backgrounds, diverse clinical comorbidities, different sexes, various developmental trajectories, and medication status is a general issue and formidable challenge, causing diverse and contradictory EEG (Kessler et al., 2016) and imaging findings of ASD (Di Martino et al., 2014; Ecker et al., 2015; Hahamy et al., 2015). Therefore, in this study, we first stratified all enrolled subjects through a data-driven approach to improve the interparticipant homogeneity in whole-brain connectivity patterns. Diseaserelated disruptions in brain networks were then assessed via case-control comparison in subgroups (Loth et al., 2015). As a result, a hypoconnected network mainly located in prefrontal and temporal areas was identified in one autistic subgroup. Specifically, functional alterations of brain areas including superior and medial temporal areas heavily involved in language processing and verbal communication (Amaral et al., 2008) were associated with social communicative deficits in this subgroup.

Cognitive flexibility deficits are proposed as potential psychological constructs underpinning major autistic domains, including restricted and repetitive behavior, atypical social interaction, and abnormal communication (Geurts et al., 2009; Lai et al., 2014). Consistent with our observation in monkeys, ASD patients are impaired in maintaining newly rewarded responses and are inclined to revert back to previously reinforced choices, which partially explains the increased severity of restricted and repetitive behaviors (Miller et al., 2015). Interestingly, MECP2 copy number positively correlated with the increased rate of regressive errors, suggesting a gene dosage-dependent severity of the phenotype in these monkeys similar to that observed in human autism (Ramocki et al., 2010). Our circuit-behavior analysis further pins down a pertinent distributed network involving the PFC (medial, orbital, and lateral parts), premotor, cingulate (anterior and rostral parts), inferior parietal, hippocampal, and parahippocampal areas, which are responsible for their performance in the reversal task. This finding is compatible with recent fMRI results in ASD patients performing a modified reversal learning task, where reduced activation was found in frontal and parietal networks that support flexible choices (D'Cruz et al., 2016). Future studies are needed to investigate the role of cognitive flexibility in autistic etiology, especially its link to social communication and repetitive behavior.

\section{Cross-species neural mappings}

We proposed a cross-species comparison strategy involving patient stratification and neural mappings from monkeys to patient subgroups. Patient stratification before cross-species mapping is an important prerequisite because within the umbrella term "ASD" there exist very different subtypes of patients in this ABIDE large repository; meanwhile, the "animal model" is not expected to resemble the human disorder in every respect (van den Heuvel et al., 2016). As such, data-driven stratification whereby multiple subgroups are biologically defined should reveal multiple networks in parallel that may underlie different symptomatic domains in autism (Loth et al., 2015). Fortunately, we found that one of two stratified subgroups demonstrated substantial overlapping abnormal brain regions with the monkey model (Fig. 7A), among which the lateral orbitofrontal cortex plays a vital role in disrupted circuits in both species (Figs. $4 C$, $6 C)$. The dysconnectivity fingerprints of these overlapping regions were similar between three TG monkeys and autistic humans, indicating that the current monkey model captured the neuropathological network features of autism to some extent. Another observation was that the transgenic monkeys exhibited both increased and decreased functional connectivity, whereas the autism subgroup 1 exhibited overall decreased functional connectivity (although the autism subgroup 2 mainly exhibited overall increased connectivity). This is consistent with the mixed findings of both hypoconnectivity and hyperconnectivity in numerous studies on autism such as one report using the ABIDE dataset (Di Martino et al., 2014). The present patient stratification strategy also supports a prior hypothesis that circuit heterogeneity stemming from individualized alterations in functional connectivity could be reduced by datadriven clustering (Hahamy et al., 2015).

This is the first neurophysiological and neuroimaging evidence reported in genetically engineered macaque monkeys, so caveats should be kept in mind. First, two subgroups of autistic patients stratified by functional brain networks did not exhibit discernible clinical or demographic differences. Although emerging evidence has demonstrated that healthy and ill individuals may share biological commonalities and form subgroups regardless of their clinical status (Marquand et al., 2016) using either behavior (Fair et al., 2012; Lombardo et al., 2016) or imaging measures (Gates et al., 2014; Costa Dias et al., 2015; Price et al., 2017), future investigation is required to address such divergence for clinical utility. Second, due to a lack of genetic data from autistic patients, we were not able to stratify patients using the same criteria as monkeys. Nevertheless, predisposition of a single genetic manipulation in animal models can be highly confined to specific neural circuits/pathways (Krol et al., 2018), which allows identification of a tangible circuit endophenotype in the animal model. This is crucial to seeking interspecies mapping to the dysfunctional brain circuits of human patients. And we observed a significant correspondence between dysconnectivity profiles between the primate genetic model and a subgroup of patients who were screened out of a large population, suggesting that either a phenotypic or genetic manifestation could converge at the circuit level (Loth et al., 2015). Another caveat is that general anesthesia was applied to all animals during EEG and MRI data collection, which has potential influence on neural synchronization and functional connectivity metrics. However, the results obtained by our animal experimental setting were not likely affected by the anesthesia conditions. Regarding the EEG results, the main EEG signature of isoflurane anesthesia is increased oscillation in lower frequency bands $(1-12 \mathrm{~Hz})$ without significant changes in the $\beta$ band (Purdon et al., 2015). As for the fMRI results, the dosage of isoflurane used in the current study is suitable for the investigation of functional brain architecture in macaque monkeys (Hutchison et al., 2014). Moreover, we took considerable caution to maintain a stable anesthesia condition in individual subjects and achieved similar anesthetic depth in both TG and WT groups. The potential effect of anesthetic agents should have been removed by contrasting the two groups. The last caveat is that rare copy number variants of MECP2 contribute only to a very small fraction of clinical cases in autism, and MECP2-related disorders such as Rett syndrome are characterized by other clinical symptoms in addition to autistic traits, which demands extra caution to appropriately interpret the present findings in terms of the complex genetic architecture of ASD. Nevertheless, this study represents an exploratory effort to identify a tangible circuit endophenotype that may serve as an intermediate bridge associating molecular genetics with dimensional symptoms or behavioral domains. 


\section{Conclusions}

MECP2 duplication-induced effect on neural connectivity of the primate model is both temporally ( $\beta$-frequency range, $12-$ $30 \mathrm{~Hz}$ ) and spatially (fronto-parieto-occipital network, prefrontal and cingulate network) dependent. Moreover, dysfunctional connectivity profiles of transgenic monkeys bear significant resemblance to those of a stratified group of autism. All of the present examinations on nonhuman primates that are conveniently adapted to human subjects not only hold crucial implications for accurate diagnosis of autism-related disorders, but also offer new insights into the development of behavioral interventions that can improve atypical locomotion and social communication.

\section{References}

Amaral DG, Schumann CM, Nordahl CW (2008) Neuroanatomy of autism. Trends Neurosci 31:137-145.

Amir RE, Van den Veyver IB, Wan M, Tran CQ, Francke U, Zoghbi HY (1999) Rett syndrome is caused by mutations in X-linked MECP2, encoding methyl-CpG-binding protein 2. Nat Genet 23:185-188.

Asaad WF, Eskandar EN (2008) A flexible software tool for temporally-precise behavioral control in Matlab. J Neurosci Methods 174:245-258.

Bernard A, Lubbers LS, Tanis KQ, Luo R, Podtelezhnikov AA, Finney EM, McWhorter MME, Serikawa K, Lemon T, Morgan R, Copeland C, Smith K, Cullen V, Davis-Turak J, Lee C-K, Sunkin SM, Loboda AP, Levine DM, Stone DJ, Hawrylycz MJ, et al. (2012) Transcriptional architecture of the primate neocortex. Neuron 73:1083-1099.

Bertero A, Liska A, Pagani M, Parolisi R, Masferrer ME, Gritti M, Pedrazzoli M, Galbusera A, Sarica A, Cerasa A, Buffelli M, Tonini R, Buffo A, Gross C, Pasqualetti M, Gozzi A (2018) Autism-associated 16p11.2 microdeletion impairs prefrontal functional connectivity in mouse and human. Brain 141:2055-2065.

Brown EN, Lydic R, Schiff ND (2010) General anesthesia, sleep, and coma. N Engl J Med 363:2638-2650.

Buckley MJ, Mansouri FA, Hoda H, Mahboubi M, Browning PG, Kwok SC, Phillips A, Tanaka K (2009) Dissociable components of rule-guided behavior depend on distinct medial and prefrontal regions. Science 325:52-58.

Chao HT, Chen H, Samaco RC, Xue M, Chahrour M, Yoo J, Neul JL, Gong S, Lu HC, Heintz N, Ekker M, Rubenstein JL, Noebels JL, Rosenmund C, Zoghbi HY (2010) Dysfunction in GABA signalling mediates autism-like stereotypies and Rett syndrome phenotypes. Nature 468:263-269.

Costa Dias TG, Iyer SP, Carpenter SD, Cary RP, Wilson VB, Mitchell SH, Nigg JT, Fair DA (2015) Characterizing heterogeneity in children with and without ADHD based on reward system connectivity. Dev Cogn Neurosci 11:155-174.

D’Cruz A-M, Mosconi MW, Ragozzino ME, Cook EH, Sweeney JA (2016) Alterations in the functional neural circuitry supporting flexible choice behavior in autism spectrum disorders. Transl Psychiatry 6:e916.

Delorme A, Makeig S (2004) EEGLAB: an open source toolbox for analysis of single-trial EEG dynamics including independent component analysis. J Neurosci Methods 134:9-21.

Di Martino A, Yan C-G, Li Q, Denio E, Castellanos FX, Alaerts K, Anderson JS, Assaf M, Bookheimer SY, Dapretto M, Deen B, Delmonte S, Dinstein I, Ertl-Wagner B, Fair DA, Gallagher L, Kennedy DP, Keown CL, Keysers C, Lainhart JE, et al. (2014) The autism brain imaging data exchange: towards a large-scale evaluation of the intrinsic brain architecture in autism. Mol Psychiatry 19:659-667.

Drysdale AT, Grosenick L, Downar J, Dunlop K, Mansouri F, Meng Y, Fetcho RN, Zebley B, Oathes DJ, Etkin A, Schatzberg AF, Sudheimer K, Keller J, Mayberg HS, Gunning FM, Alexopoulos GS, Fox MD, PascualLeone A, Voss HU, Casey BJ, et al. (2017) Resting-state connectivity biomarkers define neurophysiological subtypes of depression. Nat Med 23:28-38.

Ecker C, Bookheimer SY, Murphy DG (2015) Neuroimaging in autism spectrum disorder: brain structure and function across the lifespan. Lancet Neurol 14:1121-1134.

Fair DA, Bathula D, Nikolas MA, Nigg JT (2012) Distinct neuropsychological subgroups in typically developing youth inform heterogeneity in children with ADHD. Proc Natl Acad Sci U S A 109:6769-6774.
Fischl B, Salat DH, Busa E, Albert M, Dieterich M, Haselgrove C, van der Kouwe A, Killiany R, Kennedy D, Klaveness S, Montillo A, Makris N, Rosen B, Dale AM (2002) Whole brain segmentation: automated labeling of neuroanatomical structures in the human brain. Neuron 33:341-355.

Gates KM, Molenaar PC, Iyer SP, Nigg JT, Fair DA (2014) Organizing heterogeneous samples using community detection of GIMME-derived resting state functional networks. PLoS One 9:e91322.

Geurts HM, Corbett B, Solomon M (2009) The paradox of cognitive flexibility in autism. Trends Cogn Sci 13:74-82.

Gil-da-Costa R, Stoner GR, Fung R, Albright TD (2013) Nonhuman primate model of schizophrenia using a noninvasive EEG method. Proc Natl Acad Sci U S A 110:15425-15430.

Goldman RI, Stern JM, Engel J Jr, Cohen MS (2000) Acquiring simultaneous EEG and functional MRI. Clin Neurophysiol 111:1974-1980.

Guy J, Cheval H, Selfridge J, Bird A (2011) The role of MeCP2 in the brain. Annu Rev Cell Dev Biol 27:631-652.

Hahamy A, Behrmann M, Malach R (2015) The idiosyncratic brain: distortion of spontaneous connectivity patterns in autism spectrum disorder. Nat Neurosci 18:302-309.

Huguet G, Ey E, Bourgeron T (2013) The genetic landscapes of autism spectrum disorders. Annu Rev Genom Hum Genet 14:191-213.

Hutchison RM, Womelsdorf T, Gati JS, Everling S, Menon RS (2013) Resting-state networks show dynamic functional connectivity in awake humans and anesthetized macaques. Hum Brain Mapp 34:2154-2177.

Hutchison RM, Hutchison M, Manning KY, Menon RS, Everling S (2014) Isoflurane induces dose-dependent alterations in the cortical connectivity profiles and dynamic properties of the brain's functional architecture. Hum Brain Mapp 35:5754-5775.

Johnson WE, Li C, Rabinovic A (2007) Adjusting batch effects in microarray expression data using empirical Bayes methods. Biostatistics 8:118-127.

Judge PG, Evans DW, Schroepfer KK, Gross AC (2011) Perseveration on a reversal-learning task correlates with rates of self-directed behavior in nonhuman primates. Behav Brain Res 222:57-65.

Kessler K, Seymour RA, Rippon G (2016) Brain oscillations and connectivity in autism spectrum disorders (ASD): new approaches to methodology, measurement and modelling. Neurosci Biobehav Rev 71:601-620.

Kötter R, Wanke E (2005) Mapping brains without coordinates. Phil Trans R Soc B 360:751-766.

Krol A, Wimmer RD, Halassa MM, Feng G (2018) Thalamic reticular dysfunction as a circuit endophenotype in neurodevelopmental disorders. Neuron 98:282-295.

Lai MC, Lombardo MV, Baron-Cohen S (2014) Autism. Lancet 383:896-910.

Langfelder P, Horvath S (2008) WGCNA: an R package for weighted correlation network analysis. BMC Bioinformatics 9:559.

Liu C, Tian X, Liu H, Mo Y, Bai F, Zhao X, Ma Y, Wang J (2015) Rhesus monkey brain development during late infancy and the effect of phencyclidine: a longitudinal MRI and DTI study. Neuroimage 107:65-75.

Liu Z, Li X, Zhang J-T, Cai Y-J, Cheng T-L, Cheng C, Wang Y, Zhang C-C, Nie Y-H, Chen Z-F, Bian W-J, Zhang L, Xiao J, Lu B, Zhang Y-F, Zhang X-D, Sang X, Wu J-J, Xu X, Xiong Z-Q, et al. (2016) Autism-like behaviours and germline transmission in transgenic monkeys overexpressing MeCP2. Nature 530:98-102.

Lombardo MV, Lai M-C, Auyeung B, Holt RJ, Allison C, Smith P, Chakrabarti B, Ruigrok ANV, Suckling J, Bullmore ET, Bailey AJ, BaronCohen S, Bolton PF, Bullmore ET, Carrington S, Catani M, Chakrabarti B, Craig MC, Daly EM, Deoni SCL, et al. (2016) Unsupervised datadriven stratification of mentalizing heterogeneity in autism. Sci Rep 6:35333.

Lombardo MV, Lai MC, Baron-Cohen S (2019) Big data approaches to decomposing heterogeneity across the autism spectrum. Mol Psychiatry 24:1435-1450.

Loth E, Spooren W, Ham LM, Isaac MB, Auriche-Benichou C, Banaschewski T, Baron-Cohen S, Broich K, Bölte S, Bourgeron T, Charman T, Collier D, de Andres-Trelles F, Durston S, Ecker C, Elferink A, Haberkamp M, Hemmings R, Johnson MH, Jones EJH, et al. (2015) Identification and validation of biomarkers for autism spectrum disorders. Nat Rev Drug Discov 15:70.

Lu H, Ash RT, He L, Kee SE, Wang W, Yu D, Hao S, Meng X, Ure K, ItoIshida A, Tang B, Sun Y, Ji D, Tang J, Arenkiel BR, Smirnakis SM, Zoghbi HY (2016) Loss and gain of MeCP2 cause similar hippocampal circuit dysfunction that is rescued by deep brain stimulation in a Rett syndrome mouse model. Neuron 91:739-747. 
Lv Q, Yang L, Li G, Wang Z, Shen Z, Yu W, Jiang Q, Hou B, Pu J, Hu H, Wang Z (2016) Large-scale persistent network reconfiguration induced by ketamine in anesthetized monkeys: relevance to mood disorders. Biol Psychiatry 79:765-775.

Marquand AF, Wolfers T, Mennes M, Buitelaar J, Beckmann CF (2016) Beyond lumping and splitting: a review of computational approaches for stratifying psychiatric disorders. Biol Psychiatry Cogn Neurosci Neuroimaging 1:433-447.

Mars RB, Verhagen L, Gladwin TE, Neubert FX, Sallet J, Rushworth MF (2016) Comparing brains by matching connectivity profiles. Neurosci Biobehav Rev 60:90-97.

Mars RB, Sotiropoulos SN, Passingham RE, Sallet J, Verhagen L, Khrapitchev AA, Sibson N, Jbabdi S (2018) Whole brain comparative anatomy using connectivity blueprints. eLife 7:e35237.

McKinney WT Jr, Suomi SJ, Harlow HF (1972) Repetitive peer separations of juvenile-age rhesus monkeys. Arch Gen Psychiatry 27:200-203.

Miller HL, Ragozzino ME, Cook EH, Sweeney JA, Mosconi MW (2015) Cognitive set shifting deficits and their relationship to repetitive behaviors in autism spectrum disorder. J Autism Dev Disord 45:805-815.

Monteggia L, Heimer H, Nestler EJ (2018) Meeting report: can we make animal models of human mental illness? Biol Psychiatry 84:542-545.

Mullen T (2012) CleanLine: tool/resource info. NITRC. Available at http:// www.nitrc.org/projects/cleanline. Accessed April 9, 2018.

Neubert F-X, Mars Rogier B, Thomas Adam G, Sallet J, Rushworth MF (2014) Comparison of human ventral frontal cortex areas for cognitive control and language with areas in monkey frontal cortex. Neuron 81:700-713

Oostenveld R, Fries P, Maris E, Schoffelen J-M (2011) FieldTrip: open source software for advanced analysis of MEG, EEG, and invasive electrophysiological data. Comput Intell Neurosci 2011:156869.

Porjesz B, Almasy L, Edenberg HJ, Wang K, Chorlian DB, Foroud T, Goate A, Rice JP, O’Connor SJ, Rohrbaugh J, Kuperman S, Bauer LO, Crowe RR, Schuckit MA, Hesselbrock V, Conneally PM, Tischfield JA, Li T-K, Reich T, Begleiter H (2002) Linkage disequilibrium between the beta frequency of the human EEG and a $\mathrm{GABA}_{\mathrm{A}}$ receptor gene locus. Proc Natl Acad Sci U S A 99:3729-3733.

Price RB, Lane S, Gates K, Kraynak TE, Horner MS, Thase ME, Siegle GJ (2017) Parsing heterogeneity in the brain connectivity of depressed and healthy adults during positive mood. Biol Psychiatry 81:347-357.

Purdon PL, Sampson A, Pavone KJ, Brown EN (2015) Clinical electroencephalography for anesthesiologists: part I. Background and basic signatures. Anesthesiology 123:937-960.

Ramocki MB, Tavyev YJ, Peters SU (2010) The MECP2 duplication syndrome. Am J Med Genet A 152A:1079-1088.

Robertson CE, Ratai EM, Kanwisher N (2016) Reduced GABAergic action in the autistic brain. Curr Biol 26:80-85.

Rohlfing T, Kroenke CD, Sullivan EV, Dubach MF, Bowden DM, Grant KA, Pfefferbaum A (2012) The INIA19 template and neuromaps atlas for primate brain image parcellation and spatial normalization. Front Neuroinform 6:27.

Rubinov M, Sporns O (2010) Complex network measures of brain connectivity: uses and interpretations. Neuroimage 52:1059-1069.

Sahin M, Sur M (2015) Genes, circuits, and precision therapies for autism and related neurodevelopmental disorders. Science 350:aab3897.

Sallet J, Mars RB, Noonan MP, Neubert F-X, Jbabdi S, O’Reilly JX, Filippini N, Thomas AG, Rushworth MF (2013) The organization of dorsal frontal cortex in humans and macaques. J Neurosci 33:12255-12274.

Samaco RC, Mandel-Brehm C, McGraw CM, Shaw CA, McGill BE, Zoghbi HY (2012) Crh and Oprm1 mediate anxiety-related behavior and social approach in a mouse model of MECP2 duplication syndrome. Nat Genet 44:206-211.

Shen K, Bezgin G, Hutchison RM, Gati JS, Menon RS, Everling S, McIntosh AR (2012) Information processing architecture of functionally defined clusters in the macaque cortex. J Neurosci 32:17465-17476.

Sheskin DJ (2003) Handbook of parametric and nonparametric statistical procedures, Ed 3. Boca Raton, FL: CRC.

Shou G, Mosconi MW, Wang J, Ethridge LE, Sweeney JA, Ding L (2017) Electrophysiological signatures of atypical intrinsic brain connectivity networks in autism. J Neural Eng 14:046010.

Snyder AC, Morais MJ, Willis CM, Smith MA (2015) Global network influences on local functional connectivity. Nat Neurosci 18:736-743.

van den Heuvel MP, Bullmore ET, Sporns O (2016) Comparative connectomics. Trends Cogn Sci 20:345-361.

Van Essen DC, Glasser MF, Dierker DL, Harwell J (2012) Cortical parcellations of the macaque monkey analyzed on surface-based atlases. Cereb Cortex 22:2227-2240.

Vincent JL, Patel GH, Fox MD, Snyder AZ, Baker JT, Van Essen DC, Zempel JM, Snyder LH, Corbetta M, Raichle ME (2007) Intrinsic functional architecture in the anaesthetized monkey brain. Nature 447:83-86.

Vinck M, Oostenveld R, van Wingerden M, Battaglia F, Pennartz CM (2011) An improved index of phase-synchronization for electrophysiological data in the presence of volume-conduction, noise and sample-size bias. Neuroimage 55:1548-1565.

Wang Z, Chen LM, Négyessy L, Friedman RM, Mishra A, Gore JC, Roe AW (2013) The relationship of anatomical and functional connectivity to resting-state connectivity in primate somatosensory cortex. Neuron 78:11161126.

Whittington MA, Faulkner HJ, Doheny HC, Traub RD (2000) Neuronal fast oscillations as a target site for psychoactive drugs. Pharmacol Ther 86:171-190.

Yan CG, Zang YF (2010) DPARSF: a MATLAB toolbox for "pipeline" data analysis of resting-state fMRI. Front Syst Neurosci 4:13.

Zalesky A, Fornito A, Bullmore ET (2010) Network-based statistic: identifying differences in brain networks. Neuroimage 53:1197-1207.

Zhang Z, Cai D-C, Wang Z, Zeljic K, Wang Z, Wang Y (2019) Isofluraneinduced burst suppression increases intrinsic functional connectivity of the monkey brain. Front Neurosci 13:296. 